\title{
Image processing for hydraulic jump free-surface detection: coupled gradient/machine learning model
}

\author{
Robert Ljubičić ${ }^{1}$, Ivana Vićanović ${ }^{1}$, Budo Zindović ${ }^{1}$, Radomir Kapor ${ }^{1}$, Ljubodrag \\ Savić ${ }^{1}$ \\ ${ }^{1}$ Department of Hydraulic and Environmental Engineering, Faculty of Civil Engineering - University of \\ Belgrade, Serbia \\ E-mail: rljubicic@grf.bg.ac.rs
}

Received xxxxxx

Accepted for publication $\mathrm{xxxxxx}$

Published xxxxxx

\begin{abstract}
High-frequency oscillations and high surface aeration, induced by the strong turbulence, make water depth measurement for hydraulic jumps a persistently challenging task. The investigation of the hydraulic jump behaviour persists as an important research theme, especially with regards to the stilling basin design. Reliable knowledge of time-averaged and extreme values along a depth profile can help develop an adequate design of a stilling basin, improve safety and aid the understanding of the jump phenomenon. This paper presents an attempt of mitigating certain limitations of existing depth measurement methods by adopting a non-intrusive computer vision-based approach to measuring water depth profile of a hydraulic jump. The proposed method analyses video data in order to detect the boundary between the air-water mixture and the laboratory flume wall. This is achieved by coupling two computer vision methods: (1) analysis of the vertical image gradients, and (2) generalpurpose edge detection using a deep neural network model. While the gradient analysis technique alone can provide adequate results, its performance can be significantly improved in combination with a neural network model which incorporates a "human-like" vision in the algorithm. The model coupling reduces the likelihood of false detections and improves the overall detection accuracy. The proposed method is tested in two experiments with different degrees of jump aeration. Results show that the coupled model can reliably and accurately capture the instantaneous depth profile along the jump, with low sensitivity to image noise and flow aeration. The coupled model presented fewer false detections than the gradientbased model, and offered consistent performance in regions of high, as well as low aeration. The proposed approach allows for automated detection of the free-surface interface and expands the potential of computer vision-based measurement methods in hydraulics.
\end{abstract}

Keywords: hydraulic jump, depth measurement, stilling basins, non-intrusive measurement, computer vision, image processing 


\section{Introduction}

Reliable knowledge on flow depths and their temporal evolution is crucial for any hydraulic analysis in open channels. Free-surface profile detection and tracking, a seemingly simple research task, has proven to be rather challenging. For flow in the hydraulic jump, such measurements presents an even greater challenge due to high frequency free-surface oscillations and intense surface aeration [1]. These unfavourable conditions are present in both field and laboratory settings.

Traditional depth measuring equipment can display intrinsic unreliability and high measurement uncertainty in such hydraulic conditions. Acoustic displacement meters (ADMs; ultrasonic meters, US) are commonly used for nonintrusive hydraulic jump depth measurements. However, it was demonstrated that they are sensitive to surface aeration intensity, free-surface angle relative to the sensor, and the ejection of droplets from the air-water mixture [2]-[6]. The most significant shortcoming of the ADM depth measurement is that the acoustic beam, emitted by the transducer, penetrates the air-water mixture, i.e. it is reflected to the ADM receiver from an unknown location in the aerated region of the jump. Most researchers agree that the reflection depth is somewhere between $h_{20}$ and $h_{90}$, where $h_{\mathrm{xx}}$ is the depth at which the timeaveraged air concentration is $\mathrm{xx} \%$ [2], [4], [5]. Moreover, it was reported that the depth registered by the ADM roughly corresponds to the clear-water depth of the air-water mixture [5]. Due to this, depths acquired by the ADMs are lower than the actual depths of the air-water mixture. Such approach is also sensitive to the angle of the water surface relative to the sensor - the acoustic signal is emitted as a conical beam, so the reflection point is not necessarily in the ADM sensor axis. Finally, such approach can only provide a measurement of a small number of depths along a hydraulic jump.

In recent years, LIDAR has proven itself as a promising non-intrusive method for providing a detailed spatio-temporal description of the jump free-surface profile. However, the required equipment can be relatively expensive, and the LIDAR is reportedly unable to provide satisfactory results in regions of low surface aeration, due to the penetration of the beam below the clear-water surface (as opposed to the ADM beam which penetrates highly aerated region) [7]-[9]. Additionally, the incidence angle of the beam can have an impact on the detection results due to transmission and refraction effects [8], and the penetration depth of the LIDAR beam in the aerated flow region is yet to be adequately quantified [9]. Recent advances have also enabled the application of LIDAR for high-velocity stepped spillway flow, where it was used to quantify both flow depths and velocities [10]. However, these new insights still do not enable the use of LIDAR for both high and low aeration flow, as [10] reports an average penetration of the beam in the aerated spillway flow up to $h_{50}$ and an inability to properly detect the free-surface elevations in the clear-water flow. Despite such limitations, the potential of the LIDAR approach lies in its ability to investigate the entire aerated surface of the jump, as opposed to the single plane approach used by most imagebased methods.

The described limitations of both ADM and LIDAR approaches are significant in cases with mixed surface aeration conditions, or when the elevation of the aerated freesurface is of interest, e.g. the design of stilling basin sidewalls. In such cases, both methods are likely to underestimate the free-surface elevations used for the design of the sidewalls.

Depth measurement using electroconductivity-based phase-detection probes is still used for the purposes of hydraulic jump investigation. Although such approach is intrusive, and can be time-consuming, it can provide an estimate of the time-averaged depth profile [3]-[5], [11], [12].

Here, an important discussion is due regarding the definition of the free-surface in highly aerated flows. Research using phase-detection probes and ADMs often defines the hydraulic jump free-surface as the level at which the air concentration is $90-95 \%$. Such definitions arise in part from the nature of the measurement methods - they rely on timeaveraging of results and do not allow instantaneous detections - thus the exact position of the free-surface is not well defined. While this is a reasonable definition for many applications, some engineering tasks require additional details on the airwater mixture. For the design of stilling basin sidewalls, a more suitable definition of the free-surface would be the uppermost point of the air-water mixture, above which no water is present at the given moment. This definition implies that such point is detected instantaneously. We acknowledge that such definition is also valid only for laboratory-scale investigations.

Over the course of the last several decades, approaches based on image processing have become a popular alternative to traditional methods for monitoring hydraulic parameters such as depth, velocity, air-concentrations, etc. The appeal of image-based methods lies within their non-intrusive approach, relatively simple setup procedure, and low equipment cost. In general, several different image-based applications have been considered by researchers: (1) depth measurement/freesurface detection and tracking [2], [6], [13]-[15], (2) velocity measurements [16]-[18], (3) air concentration estimation [13], [14], etc.

For detection and tracking of the free-surface from camera recordings, several approaches are used:

1) global thresholding (i.e. single-parameter binary segmentation) based on contrast, brightness and/or colour [2], [6],

2) adaptive, local thresholding (kernel-based binary segmentation) [15], [19],

3) edge or feature detection [20]-[23],

4) temporal analysis of frame sequences [24]. 
For the purposes of free-surface detection, previous studies using image processing techniques are presented with short comments in Table 1 excluding velocimetry research.

Global thresholding methods, while simple in implementation, require high control over environmental variables such as lighting and background, and in some cases can involve tracers or dyes in order to accentuate water in captured images. Adaptive thresholding and edge detection methods are more robust in varying environmental factors but can often provide low accuracy with false detection - severe outliers that can be difficult to remove in postprocessing. Temporal analysis can be used to detect the water level based on differences between sequential images. Machine learning techniques are a relatively novel approach and are developed at a quick pace. They offer an opportunity to assess relevant visual features in an experience-based manner, similarly to the human optical mechanisms, but their complexity can vary significantly.

One of the first attempts at image-based investigation of the open channel flow was presented by Mossa and Tolve [13] for aerated hydraulic jumps in laboratory conditions. They successfully applied image processing to investigate the distribution of air-concentration in the entire hydraulic jump region. Leandro et al. [14] furthered the idea of the estimation of the amount of entrained air in air-water mixtures from pixel densities in acquired images. While innovative and nonintrusive, the authors concluded that the biggest issue of the method is the need for in-situ calibration and that results are representative only in the vicinity of the flume wall. Bung [2] used a high-speed camera at 1220 frames per second (fps) in order to investigate the free-surface roughness in strongly aerated chute flow by external image contrast enhancement and subsequent edge detection. He attempted to verify his results using an US sensor, but large discrepancy of results was found due to the penetration of the acoustic signal. Nóbrega, Schulz and Zhu [6] attempted an approach with a high-speed camera and a laser light sheet setup, while the freesurface level was detected by global thresholding.

Misra et al. [15] used a more advanced free-surface detection method based on texture segmentation by grey level co-occurrence matrices and additional postprocessing using active contours minimizing energy functionals. Yu and Hahn [19] developed an image-based method for water level monitoring based on the gradient detection, but their experiment had a noticeably small area covered by ground control points that are used for image transformations, and they were positioned far from the region-of-interest (ROI), which can cause significant extrapolation errors when transforming pixel-space to real-world coordinates. Hies et al. [20] applied their image-based detection algorithm to provide real-time water level monitoring in a small channel in Singapore. The algorithm was based on edge detection in a sharp contrast target area on the channel wall, with a subsequent application of Hough transformation to detect the straight line which represents the water level. Their results were successfully verified by radar measurements. A similar approach was later implemented for an in-situ flood warning system, improved by using an infrared projector and dedicated day and night cameras [25]. Viriyakijja and Chinnarasri [21] used Canny edge detection for laboratory flume wave depth measurements. Although the method was applied to a relatively small target area, they concluded that recordings from a camera could potentially replace wave gauge measurements with adequate accuracy, with an important quality of non-intrusiveness. While most depth/level detection methods used a fixed camera setup and assumed that no camera movement or vibrations were present during the recording period, Lin, Lin and Han [22] developed a more robust approach that could alleviate for camera movement through least-square matching and normalized crosscorrelation procedures. Ljubičić et al. [26] presented a relatively robust water level detection and tracking method for hydraulic jumps in laboratory channels, based on the gradient field analysis using large Sobel-Feldman kernels, with dedicated pre- and postprocessing phases. While the method was proven to provide satisfactory results in both high and low aeration regions of the hydraulic jump, a number of false detections were present in final results, and the overall accuracy of the method is still to be assessed.

Recent advances in image and video processing technologies have enabled high-level methods of visual perception, which can attribute meaning to the observed image features. Certain methodologies have enabled sophisticated and highly accurate general-purpose image segmentation, but large-scale reliability and accuracy are still to be achieved. These methods include those based on structured forests [27], supervised learning technologies [28]-[30], etc. These new models can significantly reinforce purpose-specific image segmentation/edge detection [30].

This paper presents an approach to image-by-image visual detection and tracking of the instantaneous free-surface of the hydraulic jump, from camera-acquired data. The aim of the research is twofold: (1) to address the aforementioned inadequacies of commonly used non-intrusive depth detection methods (namely ADM and LIDAR) with focus on mixed surface aeration conditions, and (2) to investigate the accuracy and robustness of the proposed image processing approach in both high and low aeration conditions, which are commonly present in hydraulic jumps. The aim of this research is not to provide a detailed description of the hydraulic jump at hand, but rather to investigate the applicability of image processing techniques for laboratory-scale free-surface detection and tracking.

Proposed approach is based on the fusion of results from two methods: (1) free-surface detection obtained using image gradient map analysis [26], and (2) general-purpose edge 
detection with a deep neural network (DNN) model holistically-nested edge detection (HED) [30]. Gradient analysis, as opposed to simple thresholding techniques, can be tuned to adequately capture the boundary between the water and the flume wall, even in the presence of strong image noise, spatial and temporal changes in surface aeration, and lighting conditions [26]. However, in complex, rapidly-varied conditions, gradient approach can produce a number of false detections. Thus, the free-surface detection accuracy was improved by coupling with a DNN-based edge detection phase. Proposed approach can allow for a fast and accurate spatio-temporal analysis of hydraulic jumps - estimation of depth distribution (maximum, minimum and average depths along the jump profile), frequency analysis using Fast Fourier Transform (FFT) to obtain the spectral properties of the hydraulic jump, etc. The spatial resolution of the proposed approach is considerably higher than with phase-detection probes, ADMs, and LIDAR. The method is significantly less affected by the degree of the surface aeration and provides adequate results across the entire hydraulic jump and in the downstream regions of the channel. Finally, the required equipment is more affordable than LIDAR, is easily available, and does not require training or complicated setup. In theory, there are no minimal requirements regarding camera equipment, as any high-resolution digital camera can be used for image-acquisition (including smartphone cameras).

However, the potential of the proposed approach is not to replace any of the existing non-intrusive methods, as it inevitably exhibits specific limitations of its own. It is intended to be used in conjunction with other methods to provide the best available data for specific engineering tasks.

In Section 2, all stages of the proposed detection algorithm are described: (1) experiment preparation, (2) preprocessing, (3) free-surface detection using coupled gradient/DNN-based model, (4) fusion of results, and (5) postprocessing.

In Section 3, presented methods have been applied to the camera recordings of laboratory flume hydraulic jumps. Results from proposed coupled model were compared to the previously developed gradient-only model, proposed by Ljubičić et al. [26]. It was demonstrated that the coupled model outperforms the gradient-only model in terms of detection accuracy and robustness. With the proposed coupled model, one can obtain important insight into the behaviour of hydraulic jumps.

Table 1. Summary of previous hydraulic research using image processing methods (excluding velocimetry methods)

\begin{tabular}{|c|c|c|c|c|}
\hline Paper & $\begin{array}{c}\text { Investigated } \\
\text { parameters } \\
\end{array}$ & Method & $\begin{array}{c}\text { Results dimensionality } \\
\text { (excluding time) }\end{array}$ & Comments \\
\hline $\begin{array}{c}\text { Mossa and Tolve } \\
\text { (1998) }\end{array}$ & $\begin{array}{c}\text { Air concentration } \\
\text { profile }\end{array}$ & $\begin{array}{l}\text { Principal component } \\
\text { intensity }\end{array}$ & $2 \mathrm{D}$ & $\begin{array}{c}\text { Valid only near the } \\
\text { sidewall }\end{array}$ \\
\hline $\begin{array}{c}\text { Erikson and } \\
\text { Hanson (2005) }\end{array}$ & $\begin{array}{c}\text { Wave tank level } \\
\text { detection }\end{array}$ & Edge detection & 1D & Non-aerated flow \\
\hline Misra et al. (2006) & Level & $\begin{array}{c}\text { Gray-level } \\
\text { cooccurrence matrices }\end{array}$ & $1 \mathrm{D}$ & $\begin{array}{l}\text { Valid only near the } \\
\text { sidewall }\end{array}$ \\
\hline $\begin{array}{l}\text { Yu and Hahn } \\
\text { (2010) }\end{array}$ & Level & $\begin{array}{c}\text { Sobel-Feldman } \\
\text { operator }\end{array}$ & OD & $\begin{array}{c}\text { Not applicable to } \mathrm{HJ} \text {, no } \\
\text { aeration }\end{array}$ \\
\hline $\begin{array}{l}\text { Leandro et al. } \\
\text { (2012) }\end{array}$ & $\begin{array}{l}\text { Air concentration } \\
\text { profile }\end{array}$ & Pixel densities & $2 \mathrm{D}$ & $\begin{array}{c}\text { Valid only near the } \\
\text { sidewall }\end{array}$ \\
\hline Bung (2013) & $\begin{array}{c}\text { Level + FS } \\
\text { roughness }\end{array}$ & $\begin{array}{c}\text { Single parameter } \\
\text { threholding and edge } \\
\text { detection }\end{array}$ & $1 \mathrm{D}$ & $\begin{array}{l}\text { Small ROI, valid only } \\
\text { near the sidewall }\end{array}$ \\
\hline $\begin{array}{l}\text { Nóbrega, Schulz } \\
\text { and Zhu (2014) }\end{array}$ & Level & $\begin{array}{l}\text { Single parameter } \\
\text { threholding }\end{array}$ & 1D & Valid near the sidewall \\
\hline Hies et al. (2015) & Level & Hough line transform & OD & $\begin{array}{c}\text { Not applicable to } \mathrm{HJ} \text {, no } \\
\text { aeration }\end{array}$ \\
\hline $\begin{array}{c}\text { Viriyakijja and } \\
\text { Chinnarasri (2015) }\end{array}$ & HJ profile & Canny edge detection & $1 \mathrm{D}$ & $\begin{array}{c}\text { Non-aerated flow, not } \\
\text { applicable to HJ }\end{array}$ \\
\hline Hasan et al. (2016) & Level & Hough line transform & OD & $\begin{array}{c}\text { Non-aerated flow, not } \\
\text { applicable to HJ }\end{array}$ \\
\hline $\begin{array}{c}\text { Kröhnert and } \\
\text { Meichsner (2017) } \\
\end{array}$ & $\begin{array}{c}\text { Shore-line } \\
\text { detection }\end{array}$ & $\begin{array}{c}\text { Time-lapse/motion } \\
\text { analysis } \\
\end{array}$ & $1 \mathrm{D} / 2 \mathrm{D}$ & $\begin{array}{c}\text { Potentially applicable to } \\
\text { HJ }\end{array}$ \\
\hline $\begin{array}{c}\text { Lin, Lin and Han } \\
\text { (2018) }\end{array}$ & Level & Hough line transform & OD & $\begin{array}{c}\text { Not applicable to } \mathrm{HJ}, \\
\text { resistant to camera } \\
\text { movement }\end{array}$ \\
\hline $\begin{array}{l}\text { Ljubicic et al. } \\
\text { (2019) }\end{array}$ & Level & $\begin{array}{l}\text { Sobel-Feldman vertical } \\
\text { gradients }\end{array}$ & $1 \mathrm{D}$ & $\begin{array}{c}\text { Unknown accuracy and } \\
\text { reliability }\end{array}$ \\
\hline
\end{tabular}




\section{Materials and Methods}

\subsection{Phases of free-surface detection}

In this section, we describe the two detection approaches: gradient-only and coupled gradient/HED model. The outlines of both methods can be summarized with the following steps:

1. Data preparation: splitting the video into frames and elimination of distortion (rectification) caused by the imperfections of the specific camera,

2. Detection of control points (CPs), mapping of real-world coordinates to pixel-space positions, and orthorectification of images,

3. Detection of the free-surface interface:
3.1. Using gradient analysis, with appropriate colorspace transformation and preprocessing to reduce noise and accentuate the specific features,

3.2. Using holistically-nested edge detection,

4. Fusion on the results from both detection methods,

5. Postprocessing to improve accuracy and remove false detections.

Outline of the proposed coupled model is presented in Figure 1. Sections 2.2-2.6 sequentially follow the general outline of the method.

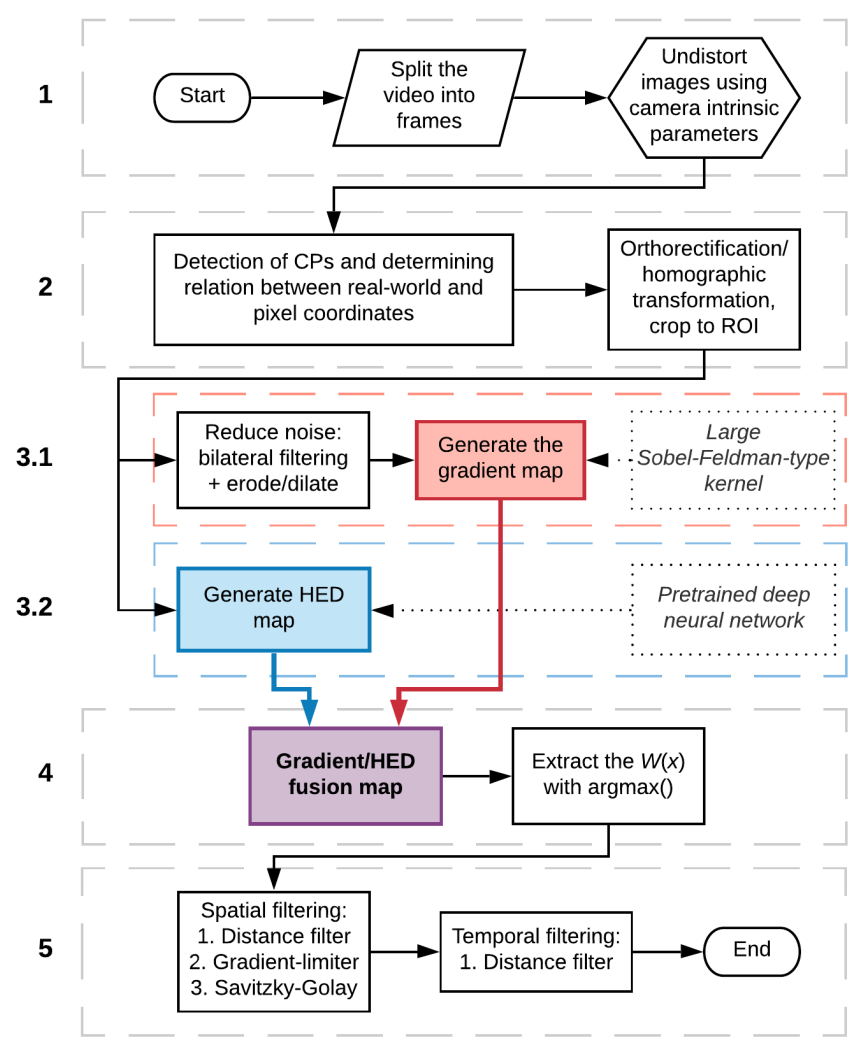

Figure 1. Outline of the proposed coupled free-surface detection model

\subsection{Camera calibration and preprocessing}

To obtain adequate results from the image data, imperfections of the camera's sensor and lens should be estimated and compensated for, prior to the actual image processing. These imperfections are intrinsic (internal) parameters and lens distortion parameters. Intrinsic parameters include focal length, principal point, and skewness coefficient. Additionally, radial and tangential distortion of the camera lens should be determined as they have a significant impact on the quality of final results. These parameters have been determined using MATLAB ${ }^{\circledR}$ Camera Calibration App [31].

Camera's extrinsic parameters, that relate the real-world 3D points (control points, $\mathrm{CPs}$ ) with previously determined locations to their in-image coordinates, depend on the actual experimental setup [32]. To obtain meaningful geometric information from camera-recorded images, relationship between pixel-space and real-world coordinates must be estimated. For this purpose, a network of wall-mounted control points (CPs) was used. In this research, CPs were specifically designed to be easily detectable in images, and with a well-defined centre point. A brightly coloured checkerboard-type pattern is used for all CPs (as seen in Figure 6). The in-image positions of the CPs can be manually determined based on a sample image. Transformation matrix between real-world and in-image coordinates of CPs was used to transform the projective perspective of the original images into an orthogonal perspective of the flume wall [26]. This procedure - orthorectification - can significantly facilitate the estimation of depths from the detected free-surface. If there are no displacements and observable vibrations of the camera and/or the ROI, the relationship between in-image and realworld $\mathrm{CP}$ coordinates can be assumed constant during the experiment. It should be noted that the CPs should be positioned so that they cover the entire ROI in which the freesurface detections will take place, and that the accuracy of the orthorectification generally increases with the increase in number of CPs.

Preprocessing stage for the gradient-based method consists of various steps to reduce the high-frequency content (visual noise) in captured images and accentuate the desired features. Image noise can be a consequence of the camera's imperfections and sensor sensitivity, but can also be caused by factors such as water droplets on the flume wall, dust, stains, etc. Since these are difficult to eliminate in large-scale experiments, the use of an appropriate noise reduction method is required. In this research, an edge-preserving bilateral filtering method was used [33]. The use of median filtering method is a good alternative, while the application of Gaussian or box (averaging) filter is undesirable since these can significantly deteriorate important visual features [33].

For the gradient analysis, the original three-channel image (usually in RGB colorspace) should be converted to an 
adequate single-channel representation. While the grayscale colorspace is predominantly used in image processing, it can be more feasible to use individual red $(\mathrm{R})$, green $(\mathrm{G})$ or blue (B) channels, or L channel of the CIELab colorspace, since those can provide more valuable information with low high frequency content. Through colorspace analysis of the captured images, it was determined for the purpose of this research that the red $(\mathrm{R})$ channel of the original RGB image has the highest signal-to-noise ratio for the laboratory setup used in this research, i.e. red channel had the lowest image noise and was used for gradient analysis in all three experiments.

Alternative to the single-channel approach - gradient analysis of the individual red $(\mathrm{R})$, green $(\mathrm{G})$ and blue $(\mathrm{B})$ channels and subsequent superposition of the results - has been determined to be inadequate as it provides unsatisfactory results in low aeration regions of the ROI.

Holistically-nested edge detection should not include a preprocessing phase, as removing information from the image can have a detrimental effect on the quality of final results. High-frequency content holds an important role in identification of edges in HED [30]. The HED was performed using all three channels of the original image to utilize as much visual information as possible.

\subsection{Gradient method}

In order to allow consistent and automated detection of the free-surface on a frame-by-frame basis, a gradient-based approach was proposed by Ljubičić et al. [26]. This approach aimed to identify the boundary between the water surface and the flume wall based on the local variability of the pixel intensity. Additionally, since the direction of the free-surface in the image is predominantly horizontal, only vertical gradient field was analysed.

Vertical gradient of any pixel neighbourhood can be determined as the first derivative of the pixel intensity field. An effective approximation of the first derivatives in horizontal or vertical direction can be obtained with a SobelFeldman operator [34]. Such operator performs a discrete linear convolution on any single-channel image $Y$, with a specific kernel $K_{y}$. Considering only vertical gradients, the Sobel-Feldman operator can be expressed as:

$$
\frac{\partial Y}{\partial y}=G_{y}=K_{y} * Y,
$$

where $*$ denotes the convolution operator. Schematic example of the Sobel-Feldman operator is presented in Figure 2. Result of this operator is a 2D array of approximated first derivatives - gradient map of the same size as the original image (nearest-pixel constant padding was used for pixel neighbourhoods near the edges). Additionally, since the detection is sensitive to the sign of the gradient, only absolute values of gradients, $\left|G_{y}\right|$, should be used in subsequent analyses.

Originally proposed kernel size by Sobel and Feldman [34] was $3 \times 3$ (Figure 2). However, such small kernel size renders the detection of gradients highly sensitive to noise and visually small features. To consistently detect larger visual features, a larger-sized Sobel-Feldman kernels must be used. For vertical gradient field, coefficients $k_{y}$ of an arbitrary sized kernel $K_{y}$ can be constructed as:

$$
\begin{aligned}
k_{y}(x, y) & =\frac{2 \Delta y}{\left|\Delta x^{a}+\Delta y^{b}\right|}, \\
\Delta x & =i-\frac{n_{x}+1}{2}, \\
\Delta y & =j-\frac{n_{y}+1}{2},
\end{aligned}
$$

where $a$ and $b$ are sensitivity coefficients, $i$ and $j$ are row and column indices, and $n_{x}$ and $n_{y}$ are the width and height of the kernel which must be odd integers. Coefficients $a$ and $b$ serve as weight distribution factors between kernel coefficients closer to the kernels centre and those farther away, and are equal to 2 in the original approach [34]. Based on experiences in [26], width of the kernel $K_{y}$ should correspond to the realworld length of $2-3 \mathrm{~cm}$, while its height should generally be higher in order to properly accentuate local vertical features without severe loss of visual information.

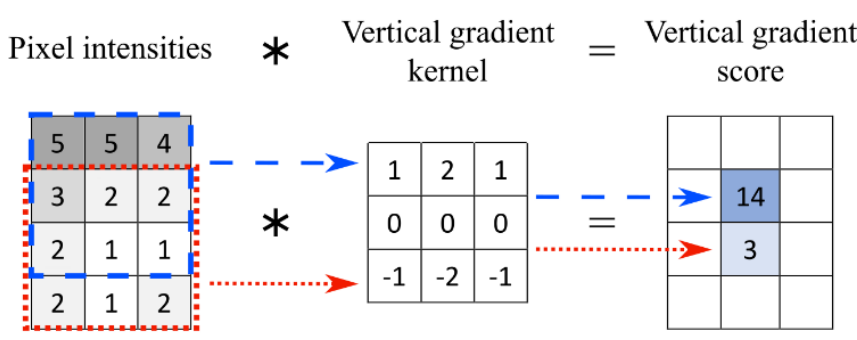

Figure 2. Schematic example of the Sobel-Feldman operator for vertical gradient estimation using $3 \times 3$-sized kernel

After the convolution step, the free-surface position can be extracted from the gradient map $\left|G_{y}\right|$, and presented as an array of positions of maximal values in each individual column $C$, i.e.:

$$
W_{G}(x)=\underset{C \subset\left|G_{y}\right|}{\arg \max }(C(x)) .
$$

\subsection{Holistically-nested edge detection}

In search of further improvement of the methodology, a state-of-the-art edge detection method is included in the algorithm - holistically-nested edge detection (HED), proposed by Xie and Tu [30]. After initial testing, HED coupled with gradient-based method was proven to deliver 
more accurate results than the gradient method alone, while decreasing the number of false detections.

Holistically-nested edge detection is based on deep learning model that utilizes fully convolutional neural networks and deeply supervised nets in order to perform image-to-image edge prediction - the algorithm does not require a sequence of images in order to obtain edge predictions. Architecture consists of a single-stream deep network with multiple side-outputs, which improve the optimization and generalization for pixel-wise classification, while providing flexibility for combined output with introduction of the fusion layer [30]. The DNN architecture ensures state-of-the-art performance in terms of edge detection and memory/time cost.

In this approach, each image was considered holistically (performing detection on the whole image simultaneously, as opposed to local approach), and the network learns features of the image from which it produces the edge predictions. Unlike some detection models, HED should not have preprocessing steps, generally does not require manual tuning of parameters, and performs well on images captured where environment and lighting conditions are potentially unknown or simply uncontrollable [30].

The HED is general-purpose detection model and its output lacks image segmentation context. However, unlike in some edge detection approaches (e.g. Canny edge detection), the result of the HED is not binary (edge/not edge). Its output is an edge probability map (Figure 3 ) where each pixel value indicates the likelihood of that pixel being a part of an edge in the original image, on a scale from 0 to $1(0$-does not belong to any edge, 1 - definitely belonging to an edge). This property allows the HED results to be superimposed with the results of other detection models to obtain a better prediction of the freesurface. In general terms, context of the detection is determined by the gradient analysis, while the HED phase/layer serves to improve the overall detection performance.

This research uses a PyTorch framework and CUDA processing for the HED stage [35]. A deep neural network is based on the Caffe network [36], which was pretrained using the Berkeley Segmentation Dataset and Benchmark [37].
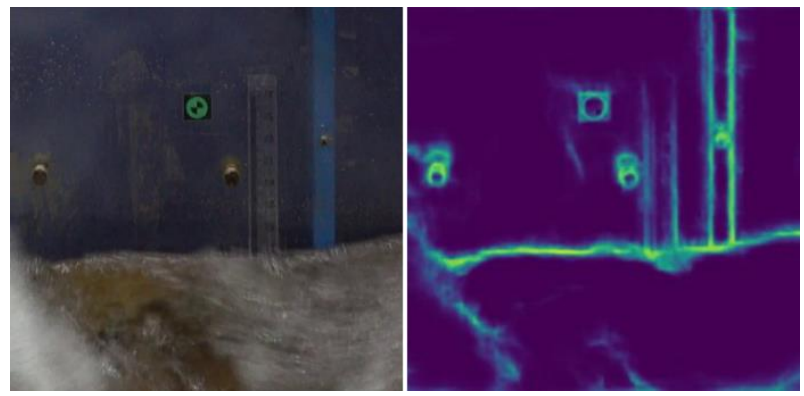

Figure 3. Left: section of the original orthorectified image; Right: example of the HED results

\subsection{Model coupling}

In order to improve the accuracy and overcome the potential unreliability of the gradient model [26], this research proposes coupling of the gradient analysis with an HED stage. The hypothesis of the coupled approach is that the superposition of results from different detection methods can lead to improvements in overall performance. Coupling of the gradient and HED results was achieved through element-wise multiplication of the individual results from gradient- and HED-based detections:

$$
F(x, y)=\left|G_{y}(x, y)\right| \cdot H E D(x, y),
$$

where $F(x, y)$ is the fusion layer (superposed) map, $G_{y}(x, y)$ is the vertical gradient map, and $\operatorname{HED}(x, y)$ is the result of the HED stage. Similar to the Eq. (3) for the gradient-based approach, the free-surface array is extracted from the fusion map $F$ as:

$$
W_{F}(x)=\underset{C \subset F}{\arg \max }(C(x)) .
$$

Examples of how the coupling of the gradient and HED results provides an improvement in detection accuracy was presented in Section 3.

\subsection{Postprocessing}

Even with coupled model, there is still a chance for detection outliers. Filtering of such outliers requires additional postprocessing steps. Two-stage filtering is recommended:

1. Spatial filtering, and

2. Temporal filtering.

2.6.1 Spatial filtering: Spatial filtering involves applying 1D filter on a free-surface array from a single timestep (single frame). A three-step procedure is proposed:

a) Distance-based filter: for any given point along the detected free-surface, a two-sided neighbourhood is selected. For that window, mean value and standard deviation are calculated. If the distance from value in the centre of the window to its mean is larger than $N$ standard deviations, that value is considered an outlier and is replaced by either the mean or median value of the window. For points near the edges of the free-surface array, nearest-value constant padding technique should be used: missing values at the end should be replaced by the last available value on that end of the free-surface array.

b) Gradient limiting filter: due to physical limitations of the open-channel flow, the vertical distance between two neighbouring points in the free-surface array cannot be arbitrarily large. For that reason, a gradient limiting filter 
is developed. For any two points in the free-surface array, maximal vertical gradient is limited to $L \times D^{m}$, where $L$ is the limiting coefficient, $D$ is the column distance between the two points (in pixels), and $m$ is the exponent in range $(0,1)$. If a value or multiple values are deemed outliers by the filter, these are replaced by linearly interpolated values between their closest nonoutlier neighbours.

c) Savitzky-Golay filter [38] is finally applied for signalto-noise ratio improvement and smoothing of the freesurface data.

2.6.2 Temporal filtering: When only steady hydraulic jumps are considered, it is reasonable to assume that, for long experiments, depth at any point along the hydraulic jump oscillates around its time-average [9]. Because of this, distance-based filter is also applied to temporal domain of each position along the free-surface.

Specific parameters for preprocessing, free-surface detection, and postprocessing used in this paper are presented in Table 2.

Table 2. Detection and filtering parameters used by both methods in both experiments

\begin{tabular}{|c|c|c|}
\hline \multicolumn{2}{|l|}{ Phase/parameter } & Value \\
\hline \multicolumn{2}{|l|}{ "Gradient analysis } & \\
\hline \multicolumn{2}{|l|}{ Colorspace [-] } & red channel \\
\hline \multicolumn{2}{|l|}{ ROI size [px] } & $1350 \times 300$ \\
\hline \multirow{4}{*}{ Sobel-Feldman kernel } & $n_{x}[\mathrm{px}]$ & 31 \\
\hline & $n_{y}[\mathrm{px}]$ & 81 \\
\hline & $a[-]$ & 2 \\
\hline & $b[-]$ & 2 \\
\hline \multicolumn{3}{|l|}{ Spatial filtering } \\
\hline \multirow{2}{*}{ Distance filter } & size $[p x]$ & 101 \\
\hline & $N[-]$ & 1.0 \\
\hline \multirow{2}{*}{ Gradient limiter } & $L[\mathrm{px}]$ & 8.0 \\
\hline & $m[-]$ & 0.8 \\
\hline \multirow[b]{2}{*}{ Savitzky-Golay } & size $[p x]$ & 11 \\
\hline & $\begin{array}{l}\text { poly. } \\
\text { order [-] }\end{array}$ & 1 \\
\hline \multicolumn{3}{|l|}{ Temporal filtering } \\
\hline \multirow{2}{*}{ Distance filter } & size [-] & 1800 \\
\hline & $N[-]$ & 5.0 \\
\hline
\end{tabular}

\subsection{Experimental setup and equipment}

Experimental setup was prepared in the Hydraulic Laboratory of the Faculty of Civil Engineering, University of Belgrade. Results from two experiments are presented in this paper:
1. Experiment 1: at discharge $Q=15.4 \mathrm{~L} / \mathrm{s}$,

2. Experiment 2: at discharge $Q=34.9 \mathrm{~L} / \mathrm{s}$.

Surface aeration conditions in the ROI were different for the two experiments, primarily as consequence of different jump lengths.

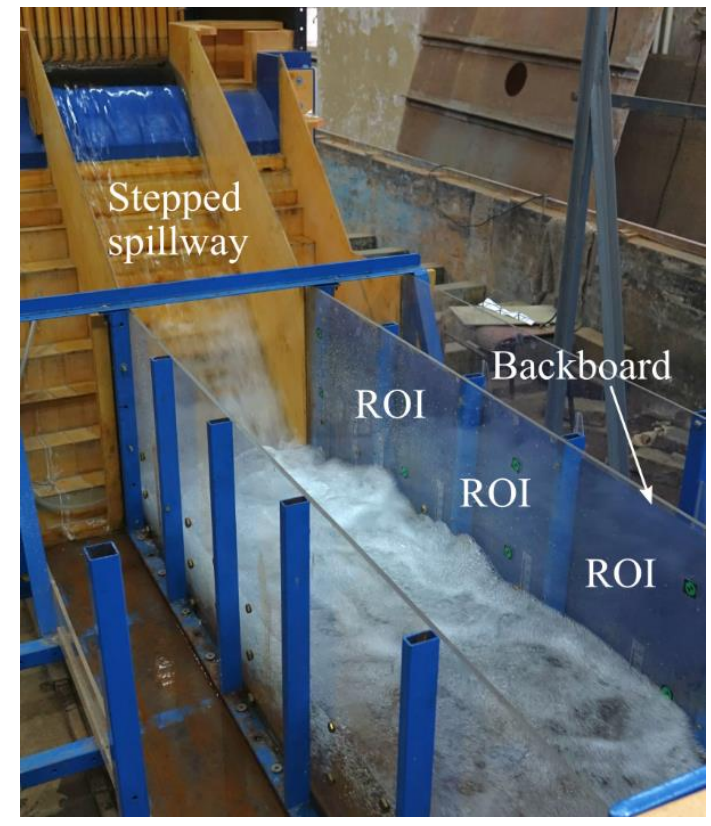

Figure 4. Laboratory setup

Experimental setup (Figure 4) consisted of a laboratory flume with an upstream stepped spillway $(0.94 \mathrm{~m}$ in height at slope $1: 1$, and $0.46 \mathrm{~m}$ in width, step height/width of $4.5 \mathrm{~cm}$ ), and a stilling basin $(2.5 \mathrm{~m}$ in length, $0.46 \mathrm{~m}$ in width). Discharge, $Q$, was measured using two sharp-crested V-notch weirs and verified with an ultrasonic transit-time flow meter. Total uncertainty of the discharge measurements was estimated to $2 \%$. Inflow conditions were held constant for 30 minutes before video recording to achieve a steady hydraulic jump. Downstream boundary condition was set with a sluice gate. Flume sidewalls were made of a transparent acrylic glass (polymethyl methacrylate, PMMA). Jump roller lengths, $L_{r}$, were measured using a procedure with light tracer particles on the free-surface and by visually tracking the position along the jump where air bubbles are rising vertically towards the freesurface, similarly to [39], [40]. Inflow conditions - clear-water $\left(h_{w 1}\right)$ and aerated depths $\left(h_{a 1}\right)$, and Froude numbers $\left(F r_{1}\right)-$ were determined according to the empirical relations presented by Boes and Hager [41], [42] for stepped spillways, and are provided for reference purposes only. It should be noted that, for the hydraulic model used in this research, the accuracy of the empirical procedure developed by Boes and Hager [41], [42] was previously investigated using phase detection probes [43]. The results indicated up to 5\% error, which is less than $1 \mathrm{~mm}$ for supercritical spillway flow. As per definition of the A-type jump, calculated inflow depths are 
located in the slopped spillway, rather than in the horizontal stilling basin.

In both experiments, A-type hydraulic jump was established [44], and the flow aeration was initialized on the stepped spillway. The reasoning behind choosing A-type instead of the classical jump is purely practical - A-type jump is more stable than classical jump in terms of jump toe oscillations. Since the aim of this research was the investigation of the detection methods, a steady A-type jump facilitated repeatability of the experiments. A bridge-type digital camera was used for video recording (Sony DSC-RX10 $\mathrm{M} 2$ ), with the resolution of $3840 \times 2160 \mathrm{px}(4 \mathrm{~K})$ at 29.97 frames-per-second (fps). Considering the high velocity turbulent flow, as well as high frequency free-surface oscillations, a large lens aperture and high shutter speeds were required to obtain good quality input data with low motion blur. A constant aperture of $\mathrm{f} / 2.8$ was used, shutter speed of $1 / 125 \mathrm{sec}$, and an ISO value (sensor sensitivity) of 400. In order to achieve adequate shutter speed with low ISO (to minimize sensor-induced image noise), two dedicated external light sources were used. Region-of-interest was identical for both experiments, and covered flume sidewall area of $135 \times 30 \mathrm{~cm}$. One-minute recordings were acquired for both experiments (1800 frames per video). Camera recording was controlled remotely using a smartphone app. During orthorectification stage, all images were scaled so that the $\mathrm{pixel} / \mathrm{mm}^{2}$ ratio is equal to 1 - area covered by one pixel corresponds to a real-world area of $1 \times 1 \mathrm{~mm}$. Such scaling significantly reduced computational complexity of the algorithm, while allowing simple extraction of data from processed images. Dark blue backboard was also placed behind the ROI to enhance contrast and reduce reflections and visual noise from the background. Any residual reflections off the flume wall were filtered in the preprocessing stage. Compared to the available research, the proposed methods enabled better longitudinal spatial resolution for instantaneous detections ( $0.1 \mathrm{~cm}$ after downscaling) than ADM (up to $15 \mathrm{~cm}$ in [5]) and LIDAR (up to $0.8 \mathrm{~cm}$ in [8]) for similar interrogation lengths in the stilling basins.

Regarding the position of the camera relative to the ROI, two approaches were considered (Figure 5):

a) Recording of the wall surface closer to the camera - inlevel configuration, and,

b) Recording of the opposing wall surface - overhead configuration.

While the in-level configuration (Figure 5a) is somewhat easier to setup, three things are to be considered. Firstly, the thickness of the flume wall can cause light refraction and distort the detected free-surface inside the flume. The extent of refraction-induced errors depends on the wall material and its thickness. Also, the distance between the plane in which the CPs are mounted and the actual ROI plane is an additional source of errors. Secondly, since the aeration in the jump varies with depth, additional noise can appear in the image which increases the undesirable high-frequency content. Such noise may require additional filtering steps. Thirdly, the camera must always be positioned in such a way that it records only the free-surface on the flume wall closest to the camera to avoid false detections from the background.

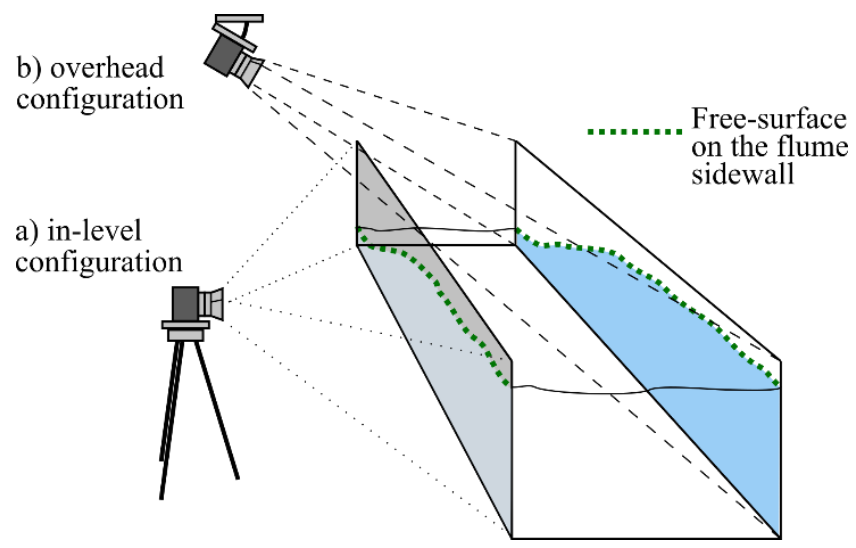

Figure 5. Camera configurations relative to the ROI

Considering the aforementioned issues, overhead camera configuration (Figure $5 b$ ) was chosen for both experiments. Camera was aimed towards the centre of the ROI and was oriented perpendicularly to the flume wall (in the horizontal plane). The effect of the camera positioning in relation to to the ROI on the final results has not been investigated for the purpose of the current paper.

For orthorectification purposes, a total of $12 \mathrm{CPs}$ were positioned relatively equidistantly into 3 rows and 4 columns so that all detections are carried out inside the CP-covered area, and the orthorectification error is minimized. Since the camera position was constant during the experiments, position of the CPs was determined for empty channel before the actual experiments and verified post-experimentally when the channel was again empty of water. The real-world position of the CPs was determined from distance measurements using least-square adjustment [45]. The relationship between the real-world and in-image coordinates of CPs was determined using least-square homographic transformation with random sample consensus method (RANSAC) [46].

At the moment, there is no specific calibration procedure for finding optimal method parameters, and the calibration process is based on experience and trial-and-error. However, as shown in Section 3, one set of parameters covers a wide range of discharges and aeration intensities, and there are no requirements for recalibration of parameters in a single flume. For both experiments, identical set of preprocessing and postprocessing parameters was used (Table 2), and detection was performed on orthorectified images obtained with the same set of intrinsic and extrinsic camera parameters.

Information on hydraulic conditions during the experiments is presented in Table 3 . 
In order to obtain an assessment of the accuracy for both models, manual reference depth measurements were taken from images at predefined stations using wall-mounted gauges (Figure 6). Reference measurements were obtained as average visual observations of the free-surface levels from 3 independent examinees, similarly to [15] (with regards to freesurface definition from the Introduction). Reference measurements were taken at $t=\{0,10,20,30,40,50,60\}$ seconds from the beginning of the video, at seven stations along the jumps where $x=\{11.4,19.5,27.5,40.4,66.9,97.9$, $127.4\} \mathrm{cm}$.

Finally, to relate the performance of the proposed approach to more traditional methods, depth measurements were performed using an acoustic displacement meter (HC-SR04) at the seven reference stations. Sampling frequency of $\sim 43 \mathrm{~Hz}$ was used, and temperature compensation of sound speed was implemented. ADM acquisition was performed at the flume centerline for 1 minute at each station, and the mean depth was estimated by time-averaging without raw data filtering. Maximal depths were also extracted from the raw data. The ADM was unable to detect minimal depths as acoustic signal would occasionally become trapped in the air-water mixture for a random amount of time - thus the ADM would report negative depths. Here it should be noted that the presented comparison is for reference purposes only, as the image processing and ADM data were taken at different times and locations in the flume (sidewall vs. centreline). The comparison serves to indicate the potential inadequacy of the ADM approach for the design of the stilling basin sidewalls, as ADM is unable to record the free-surface levels in the vicinity of the sidewalls.

Table 3. Hydraulic conditions during the experiments

\begin{tabular}{|c|c|c|c|c|c|}
\hline \multirow[b]{2}{*}{ Exp. } & \multirow[b]{2}{*}{$\begin{array}{c}\text { Discharge } \\
Q \\
{[\mathrm{~L} / \mathrm{s}]} \\
\end{array}$} & \multirow{2}{*}{$\begin{array}{c}\text { Froude } \\
\text { number } \\
F r_{1} \\
{[-]}\end{array}$} & \multirow[b]{2}{*}{$\begin{array}{c}L_{r} \\
{[\mathrm{~cm}]}\end{array}$} & \multicolumn{2}{|c|}{ Inflow depth } \\
\hline & & & & $\begin{array}{c}\text { clear- } \\
\text { water } h_{w 1} \\
{[\mathrm{~cm}]}\end{array}$ & $\begin{array}{c}\text { aerated } \\
\boldsymbol{h}_{a 1} \\
{[\mathrm{~cm}]}\end{array}$ \\
\hline$\overline{c 1}$ & 15.4 & 8.2 & ב56 & 1.2 & 2.7 \\
\hline 2 & 34.9 & 7.3 & 82 & 2.2 & 4.4 \\
\hline
\end{tabular}

\section{Results and discussion}

In this section, image processing methods are applied to free-surface detection and subsequent extraction of depth profiles in two experiments with different discharge rates and jump lengths. Following results are presented:

1. Examples of results from both detection models,

2. Accuracy analyses/error estimates, using visual reference data,

3. Comparison of time-averaged and maximal depth profiles for both experiments, obtained using image processing and ADM,
4. Standard deviations of free-surface levels in the ROI,

5. Investigation on how the coupling of two models improves the overall accuracy,

6. Spectral analyses,

7. Repeatability analysis.

Examples of detected free-surface profiles using gradient and coupled model are presented in Figure 6 for both experiments, along with their respective jump roller lengths $L_{r}$. The relative roller lengths $L_{r} / h_{2}$ (where $h_{2}$ is the downstream sequent depth) were 4.3 and 3.9 for the two experiments, which is in general agreement with the observations of Peterka for classical hydraulic jumps where $L_{r} / h_{2}$ is between 4 and 6 , and that such ratio increases with an increase in Froude numbers. The example frames in Figure 6 were intentionally chosen so that they demonstrate some of the characteristic false detections of the gradient model. They illustrate the main conclusions regarding the results from two models:

1. Accuracy of both models is comparable across most of the ROI. Both models adequately describe the freesurface, with some smaller differences,

2. Coupled model detects somewhat higher depths across a majority of the ROI,

3. Coupled model is more sensitive to detection of sudden splashes, as demonstrated by the details 1 and 4 ,

4. Gradient-only model is more susceptible to false detections of the free-surface levels in conditions of spatially varying surface aeration intensity (details 1-5 in Figure 6).

Depths obtained using both image processing models are presented against manually acquired reference values in Figure 7, and the average differences between detected and reference values at the seven gauging stations are presented in Table 4. Primary aim of this comparison is investigation of accuracy and (non-)uniformity of free-surface perception of both models, relative to the human perception. The results in Figure 7 and Table 4 indicate that the gradient model generally perceives lower depths than human eye across the entire ROI by as much as $1.6 \mathrm{~cm}$ on average at certain stations. Such underestimations are more prominent in the upstream low depth/high aeration regions of the ROI (below $15 \mathrm{~cm}$ in Figure $7)$. The coupled model shows better agreement with reference values across the ROI, as demonstrated in Figure 7 and Table 4. Average differences at the gauging locations for the gradient model were -1.02 and $-0.89 \mathrm{~cm}$ for the experiments 1 and 2, respectively. For the coupled model, these differences were reduced to -0.07 and $-0.04 \mathrm{~cm}$, respectively. The performance of the gradient model improves in the high depth/low aeration regions, while the performance of the coupled model is more uniform across the ROI (as shown in Figure 7 and Table 4). 


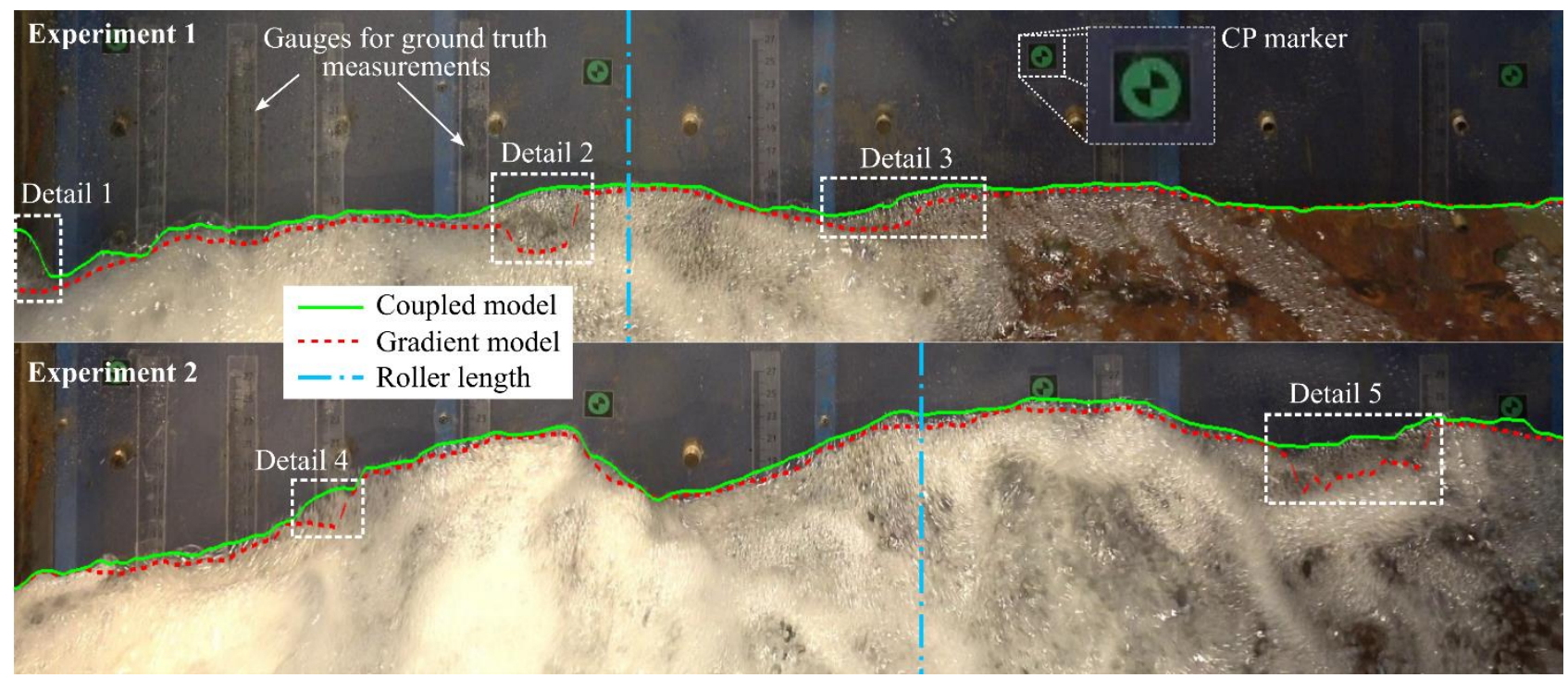

Figure 6. Example of results from both experiments, with several false detections of the gradient model highlighted

Table 4. Absolute differences between detected and visual reference values, collected at seven stations along the jump

\begin{tabular}{|c|c|c|c|c|c|c|c|c|c|}
\hline \multirow[b]{2}{*}{ Exp. } & \multirow[b]{2}{*}{ Model } & \multicolumn{7}{|c|}{ Difference } & \multirow{2}{*}{$\begin{array}{c}\text { Average } \\
{[\mathrm{cm}]}\end{array}$} \\
\hline & & $\begin{array}{c}W\left(x_{1}\right)-Z_{1} \\
{[\mathbf{c m}]}\end{array}$ & $\begin{array}{c}W\left(x_{2}\right)-Z_{2} \\
{[\mathrm{~cm}]}\end{array}$ & $\begin{array}{c}W\left(x_{3}\right)-Z_{3} \\
{[\mathbf{c m}]}\end{array}$ & $\begin{array}{c}W\left(x_{4}\right)-Z_{4} \\
{[\mathrm{~cm}]}\end{array}$ & $\begin{array}{c}W\left(x_{5}\right)-Z_{5} \\
{[\mathrm{~cm}]}\end{array}$ & $\begin{array}{c}W\left(x_{6}\right)-Z_{6} \\
{[\mathrm{~cm}]}\end{array}$ & $\begin{array}{c}W\left(x_{7}\right)-Z_{7} \\
{[\mathbf{c m}]}\end{array}$ & \\
\hline \multirow{2}{*}{1} & Gradient & -1.46 & -1.59 & -1.19 & -1.01 & -1.04 & -0.58 & -0.23 & -1.02 \\
\hline & Coupled & -0.24 & -0.19 & 0.01 & 0.09 & -0.17 & 0.10 & -0.07 & -0.07 \\
\hline \multirow{2}{*}{2} & Gradient & -1.43 & -1.32 & -0.53 & -0.93 & -0.86 & -0.64 & -0.53 & -0.89 \\
\hline & Coupled & -0.39 & -0.12 & 0.23 & 0.07 & -0.06 & 0.07 & -0.06 & -0.04 \\
\hline
\end{tabular}

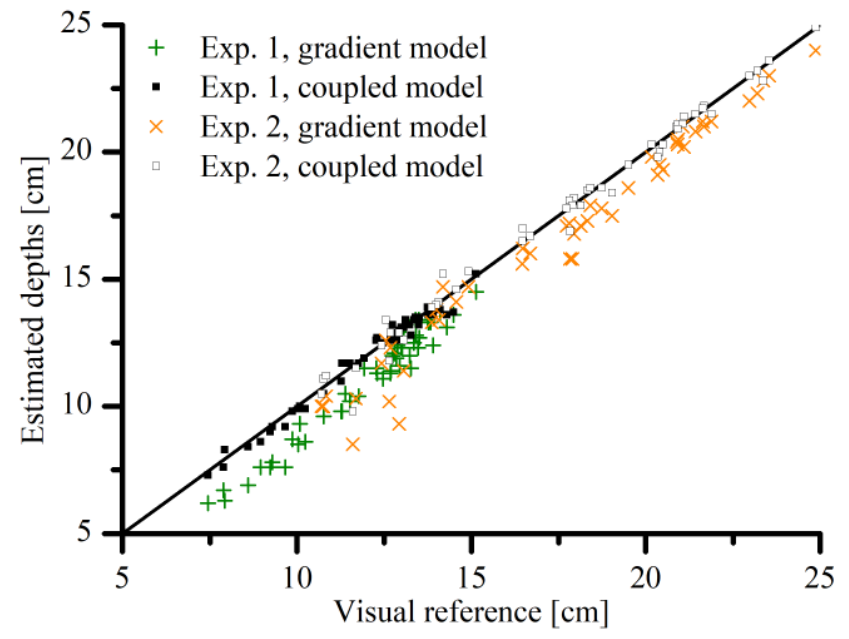

Figure 7. Comparison of results from two models against reference values for both experiments

The accuracy of both detection models is also evaluated, and the results are presented in Table 5 in terms of: (1) root mean-square error (RMSE) relative to the visual reference values, (2) coefficient of determination $R^{2}$, and (3) linear fit parameters (slope and intercept). The error estimates indicate that the coupled model outperforms the gradient model in terms of RMSE by $313 \%$ in the first experiment and $163 \%$ in the second (relative to the manually acquired reference values). Variance of results from both methods is similar, as indicated by the $R^{2}$, and is only around $2 \%$ higher for the coupled model. This suggests a similar contribution of random errors in the results of both models. To estimate the contribution of systematic errors, linear fit regression was determined for both models - a linear fit with slope of 1 and intercept value of 0 would indicate an absence of systematic errors. Linear regressions of gradient model results indicate a systematic underestimation of reference values in the high aeration regions of the jump, as shown by the negative intercept of -2.8 and $-1.5 \mathrm{~cm}$, and a regression slopes higher than 1 in both experiments. Results from the coupled model produce a better linear model, with slope value close to 1 and intercept close to 0 . Figures 8 and 9 present time-averaged and maximal depths along the jump profile for experiments 1 and 2 , respectively, along with time-averaged and maximal depths at seven reference stations obtained using ADM. The results confirm the previously recognized differences between the results obtained with the two models. An examination of the average depth profiles indicates that the difference between the two models is as high as 1.5 and $1.1 \mathrm{~cm}$, with an average 
difference across the entire jump profile of 0.7 and $0.8 \mathrm{~cm}$ for experiments 1 and 2, respectively. The coupled model is persistently detecting higher free-surface levels across the entire jump profile. The difference in results generally reduces in the downstream direction as the surface aeration intensity decreases. The envelope of maximal values is similarly shaped but higher depths are evident in the case of the coupled model, by $0.6 \mathrm{~cm}$ on average in both experiments.

Table 5. Statistical indicators of models' performances

\begin{tabular}{c|c|c|c|c|c}
\multirow{2}{*}{ Exp. } & \multirow{2}{*}{ Model } & $\begin{array}{c}\text { RMSE } \\
{[\mathbf{c m}]}\end{array}$ & $\begin{array}{c}\boldsymbol{R}^{\mathbf{2}} \\
{[-]}\end{array}$ & $\begin{array}{c}\text { Linear fit } \\
\text { Slope } \\
{[-]}\end{array}$ & $\begin{array}{c}\text { Intercept } \\
{[\mathbf{c m}]}\end{array}$ \\
\hline \hline \multirow{2}{*}{1} & Gradient & 1.13 & 0.964 & 1.147 & -2.78 \\
& Coupled & 0.27 & 0.981 & 1.003 & 0.10 \\
\hline \multirow{2}{*}{2} & Gradient & 1.16 & 0.970 & 1.034 & -1.49 \\
& Coupled & 0.44 & 0.988 & 0.999 & -0.02
\end{tabular}

Time-averaged depths obtained using ADM in experiment 1 show better agreement with the results of the gradient model, with an average absolute difference of $0.5 \mathrm{~cm}$, while the average absolute difference between the coupled model and ADM results is $0.6 \mathrm{~cm}$. As discussed in the Introduction, the $\mathrm{ADM}$ is expected to indicate depths between $h_{20}$ and $h_{90}-$ lower than time-averaged instantaneous depths due to the penetration of the acoustic beam in the air-water mixture. This difference is the lowest in the upstream region of the ROI, where the slope of the free-surface is the highest - the conical acoustic beam is likely not reflected off a point vertically bellow the sensor, but rather from an unknown point further downstream. Such differences also decrease in the downstream direction with the reduction of the surface aeration intensity.

For experiment 2, the average difference between the ADM and the gradient model is around $0.6 \mathrm{~cm}$, and $1.3 \mathrm{~cm}$ for the coupled model. As with the results of experiment 1, such differences decrease in the downstream direction with the reduction of surface aeration intensity.

However, differences are evident when maximal detected depths are considered. For experiment 1 , gradient and coupled model indicate maximal depths in the ROI of 17.1 and $17.5 \mathrm{~cm}$, respectively, while the ADM results show a maximum of $15.8 \mathrm{~cm}$. The ratio of maximal depth in the ROI and time-averaged depths at the furthest downstream point in the ROI (hereafter maximal-to-downstream depth ratio) is 1.33 and 1.35 for the gradient and coupled models, respectively. For ADM results, such ratio is 1.23 . This ratio is an important parameter for the stilling basin sidewall design. Differences in maximal detected depths, obtained using different methods, decrease in the downstream direction with the reduction of the surface aeration intensity.

For experiment 2, maximal detected depths using gradient and coupled models are 26.8 and $27.4 \mathrm{~cm}$, with maximal-to- downstream depth ratios of 1.28 and 1.29 , respectively. ADM results indicate a maximum of $24.4 \mathrm{~cm}$, and maximal-todownstream ratio of 1.21 . As in the experiment 1 , differences between maximal depths decrease in the downstream direction.

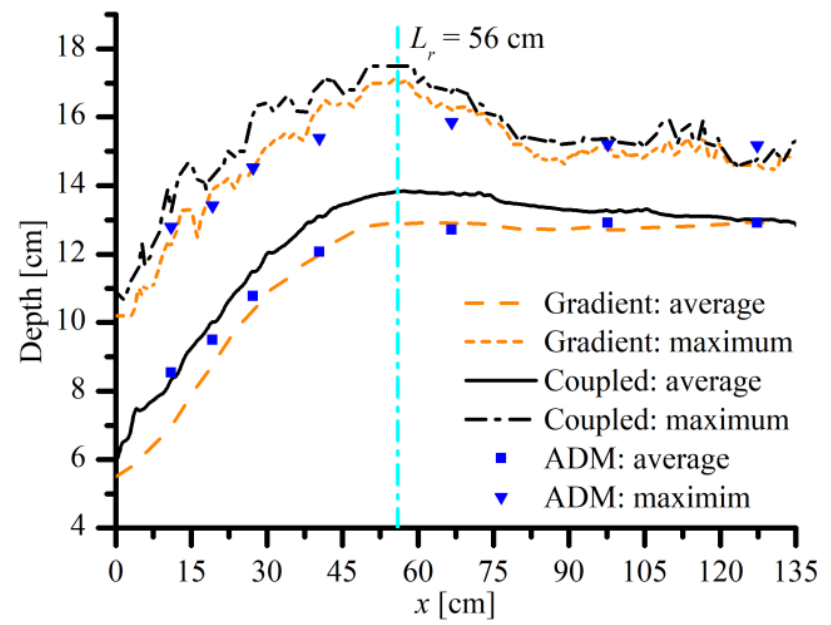

Figure 8. Average, maximal and minimal depth profiles of the experiment 1

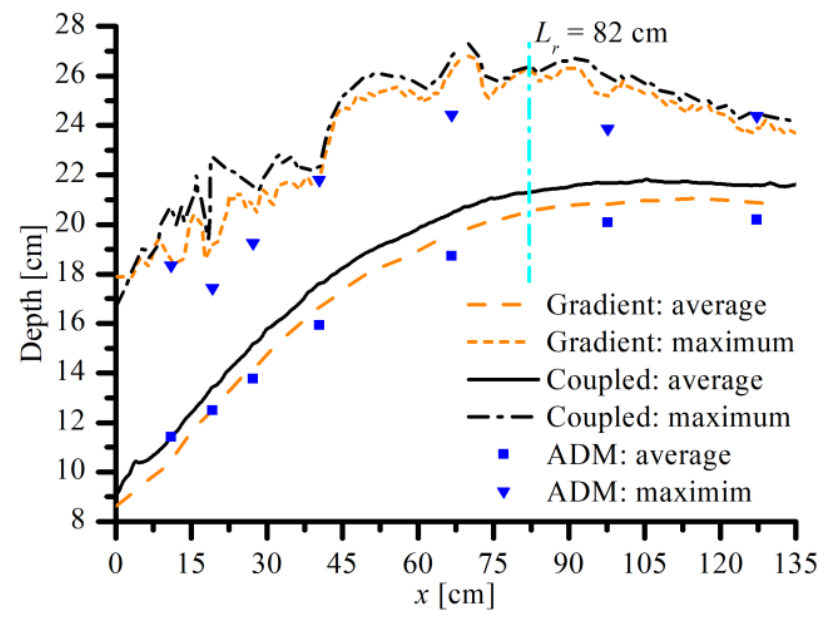

Figure 9. Average, maximal and minimal depth profiles of the experiment 2

Similar conclusions regarding the relationship of imagedetected and ADM results have been presented by [6] for classical jumps - depths obtained using ADM were consistently lower than those from the image analysis, and that such differences were decreasing in the downstream direction. The ADM results are in better agreement with the gradientonly model across the entire ROI in both experiments. In experiment 1 for $x>30 \mathrm{~cm}$, such differences are less than $1 \mathrm{~mm}$ on average. However, since Chachereau and Chanson [5] state that the ADM roughly detects the clear-water depth in the aerated regions of the hydraulic jump, this result can substantiate the previous conclusion that the gradient-only model exhibits a slight tendency for underestimation of visually detected free-surface levels. This should be taken as a limitation in cases where the detection of the visual free- 
surface is critical, such as for the design of stilling basin sidewalls. However, the differences between the gradient and the coupled model are lower than between the two imagebased approaches and ADM. Although it contains a higher percentage of false detections, the gradient model still presents valuable results for the hydraulic research of stilling basins when the quality of the raw data is adequate.

In addition to time-averaged and maximal depths, standard deviations of free-surface levels, $\sigma_{h}$, are presented in Figures 10 and 11. Standard deviations from time-averaged depths decrease steadily and monotonically in the downstream direction, with both models showing relatively consistent results. Relative to the clear-water inflow depths $h_{w 1}$, the maximal standard deviations are approx. $1.4 h_{w 1}$, and $1.2 h_{w 1}$, while the minimal standard deviations are $0.5 h_{w 1}$ and $0.4 h_{w 1}$, for the experiments 1 and 2 respectively. This is in general agreement with the experimental results from Li et al. [9] for similar Froude numbers. Similarly as in [9], the ratio of standard deviations to $h_{w 1}$ are decreasing with the decrease in inflow Froude numbers $F r_{1}$.

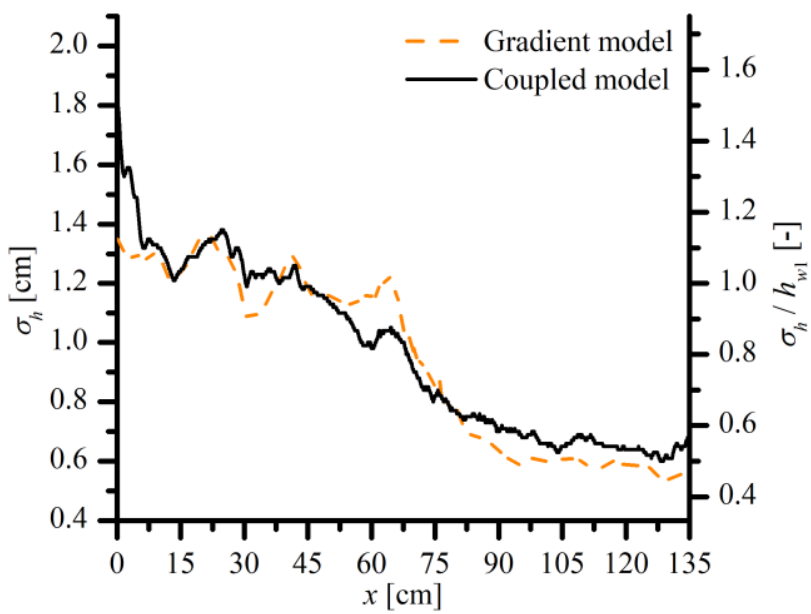

Figure 10. Standard deviations of free-surface levels in the ROI, experiment 1

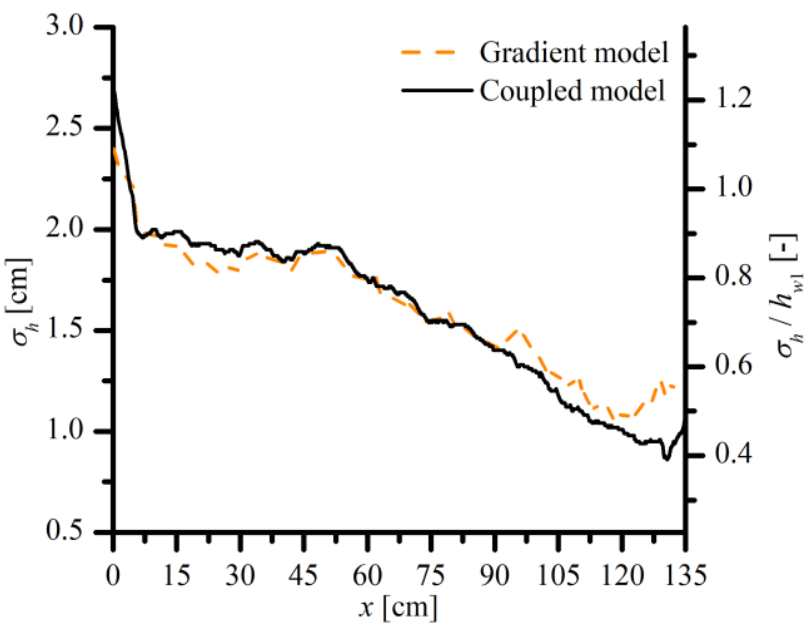

Figure 11. Standard deviations of free-surface levels in the ROI, experiment 2
For a closer examination of how the coupled model reduces the number of false detections and improves accuracy compared to individual gradient- and HED-based models, results from three characteristic columns $(x=\{29.6,48.4$, $130.7 \mathrm{fm}$ ) from a sample image were analysed (Figure 12). For each selected column, individual gradient and HED, and superposed results were shown, along with the manually acquired reference value. According to Eqs. (3) and (5), inimage position of the free-surface at station $x$ along the jump is determined by the position of the maximal value in that column in the fusion map $F(x, y)$. Gradient and HED results used for this examination are taken before the final filtering in the postprocessing phase.

Figure 13 shows the case which demonstrates that the HED, in general, does not differentiate between horizontal and vertical edges. Such vertical edges can cause ambiguity in HED results in their vicinity. However, the gradient analysis stage incorporates a "horizontal bias" due to fact that only vertical gradients are analysed, which improves the overall performance of the coupled model. The absolute difference between the detected and visual reference value, before the final filtering steps in the postprocessing stage, is $0.2 \mathrm{~cm}$.

In Figure 14, the results of the gradient model are polymodal with two dominant peaks. While the maximal value in the column of the gradient map is falsely detected around $h=8.2 \mathrm{~cm}$, the HED model accurately detects the true free-surface position in the image. The second highest peak of the gradient map coincides with maximal value in the HED map, and the resulting superposed map $F$ shows a water level at $0.1 \mathrm{~cm}$ from the visual reference value.

Figure 15 shows that the results from HED map can be polymodal in cases of background noise. The results of the gradient analysis are more resistant to the presence of background features smaller than the size of the convolution kernel $K_{y}$. and they show maximal gradients significantly closer to the reference value. This also shows that the kernel $K_{y}$ acts as a spatial filter. Superposition of results from both methods successfully deals with the false peak of the HED around $h=10.6 \mathrm{~cm}$, and the position of the maximal value in fusion map $F$ coincides with the visual reference value.

Results shown in Figures 13-15 provide an important insight into the mechanisms which enable the high accuracy and robustness of the coupled free-surface detection model. In general, the HED map serves as a weight function on top of the context-specific results of the gradient analysis. This complementary effect reduces both the uncertainty of the detections and the required filtering efforts in the postprocessing stage. 


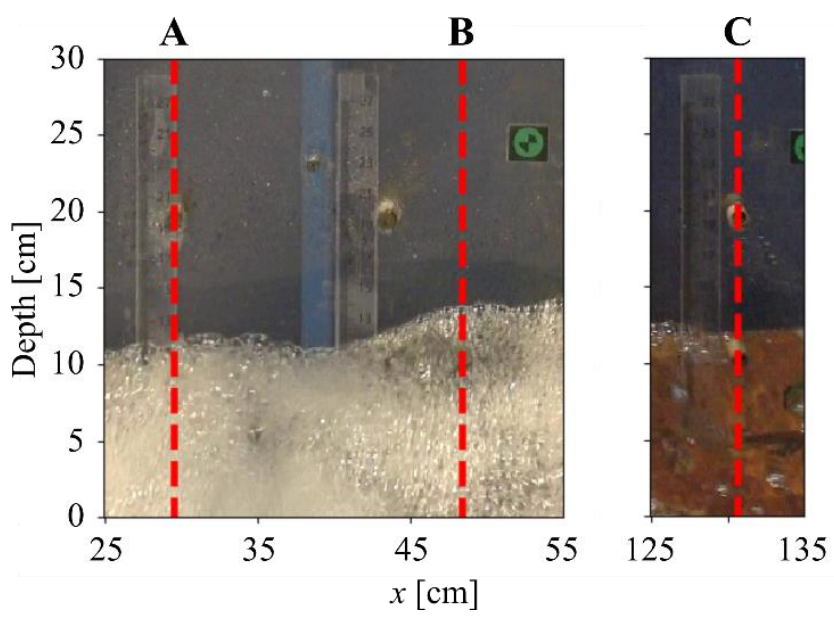

Figure 12. Columns for comparison of detection models

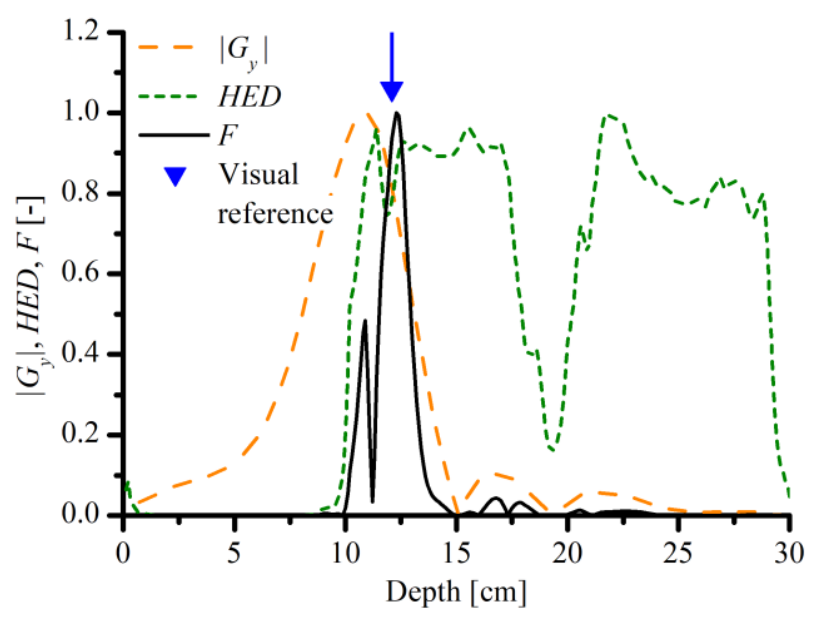

Figure 13. Comparison of detection scores at $x=29.6 \mathrm{~cm}$ (column A in Figure 12)

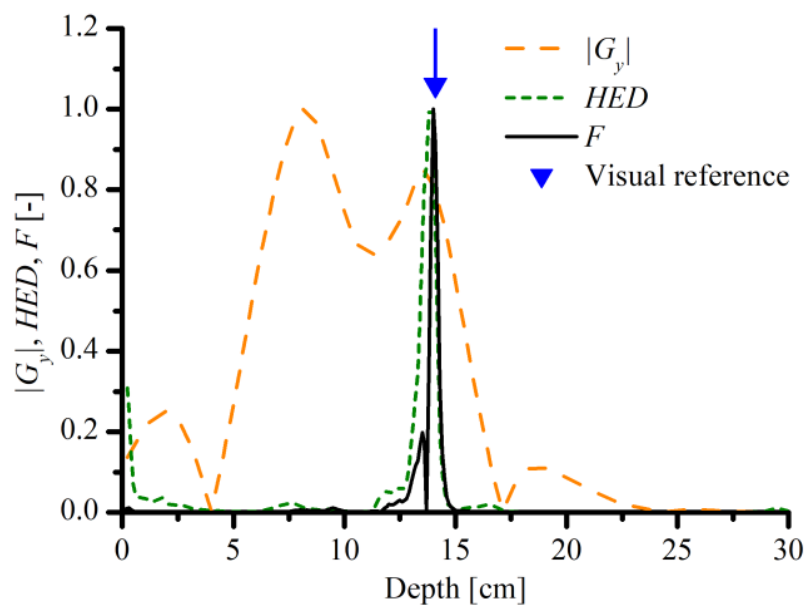

Figure 14. Comparison of detection scores at $x=48.4 \mathrm{~cm}$ (column B in Figure 12)

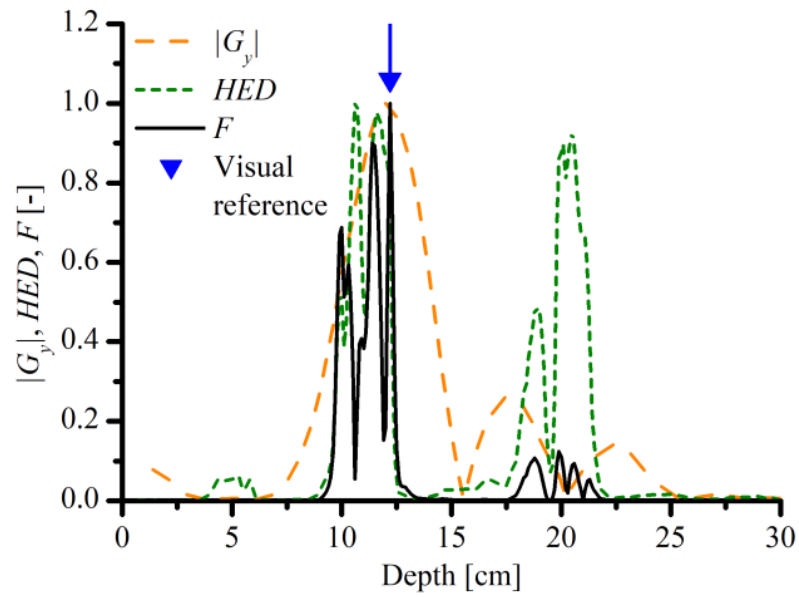

Figure 15. Comparison of detection scores at $x=130.7 \mathrm{~cm}$ (column $\mathrm{C}$ in Figure 12)

Frame-by-frame approach of the proposed image processing methods allow for detailed spectral analyses of the hydraulic jump behaviour through fast Fourier transformation (FFT). Because the free-surface level is tracked in a significantly higher number of points along the hydraulic jump than it could be possible with an ADM, FFT spectrums can be obtained for any station along the jump. This can significantly facilitate the processing of large amounts of data, to obtain a better insight into the hydraulic jump behaviour.

Figures 16 and 17 present such spectrums for 4 stations relative to the jump roller lengths, where $x / L_{r}=\{0.2,0.5,1.0$, $1.5\}$. Spectrums of both methods are comparable, and show dominant oscillating frequencies of $\sim 2.2 \mathrm{~Hz}$, which is in general agreement with previous research [8]. However, the station $x / L_{r}=0.5$ has a somewhat higher number of significant frequencies relative to the other three stations, in both experiments. At stations $x / L_{r}>1$, oscillation amplitudes decrease significantly for all analysed frequencies.

Finally, to examine the repeatability of the results of the proposed coupled model, four additional 1-minute recordings were made for both experiments. Time-averaged depth profiles of four repeated tests, along with the original results from Figures 8 and 9, are presented in Figures 18 and 19. The results indicate a significant repeatability of the results from the proposed coupled model. The average absolute difference between the five profiles and the global time-averaged profile (mean profile of five tests) is $0.05 \mathrm{~cm}$ for the conditions of experiment 1 , and $0.08 \mathrm{~cm}$ for the conditions of experiment 2 . Such differences are the most prominent in the upstream region of the ROI where the free-surface fluctuations are the highest. 

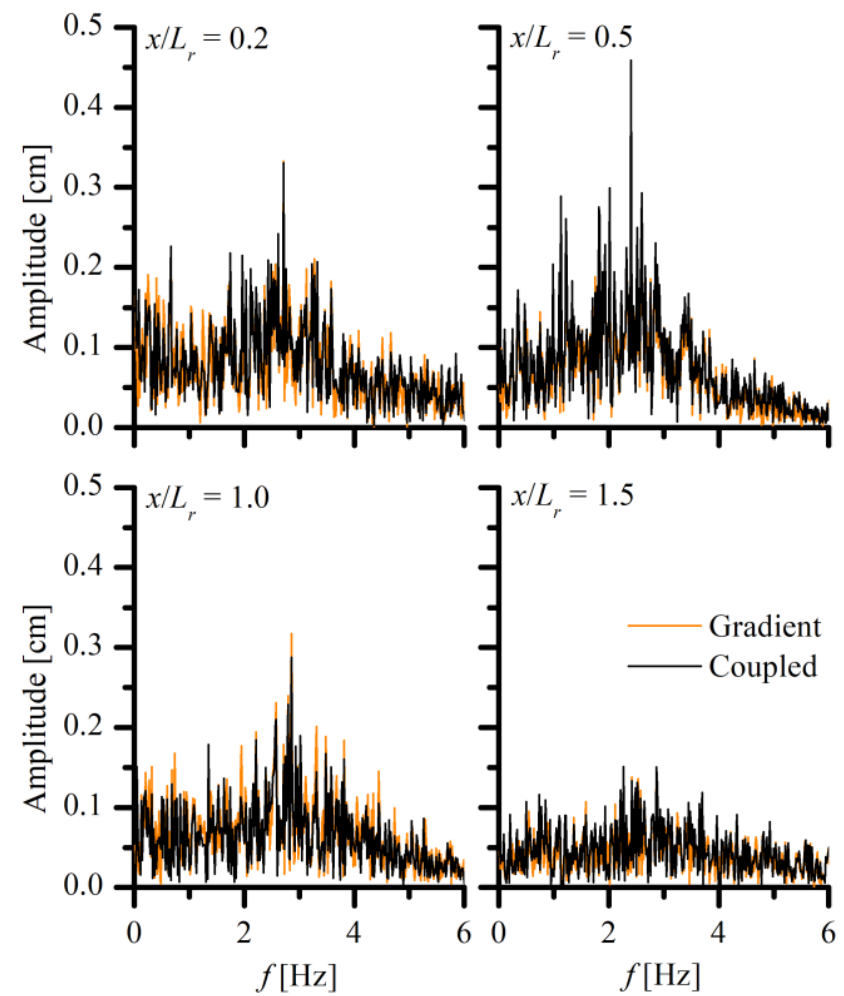

Figure 16. FFT spectrums of free-surface oscillations for four stations in the ROI, experiment 1
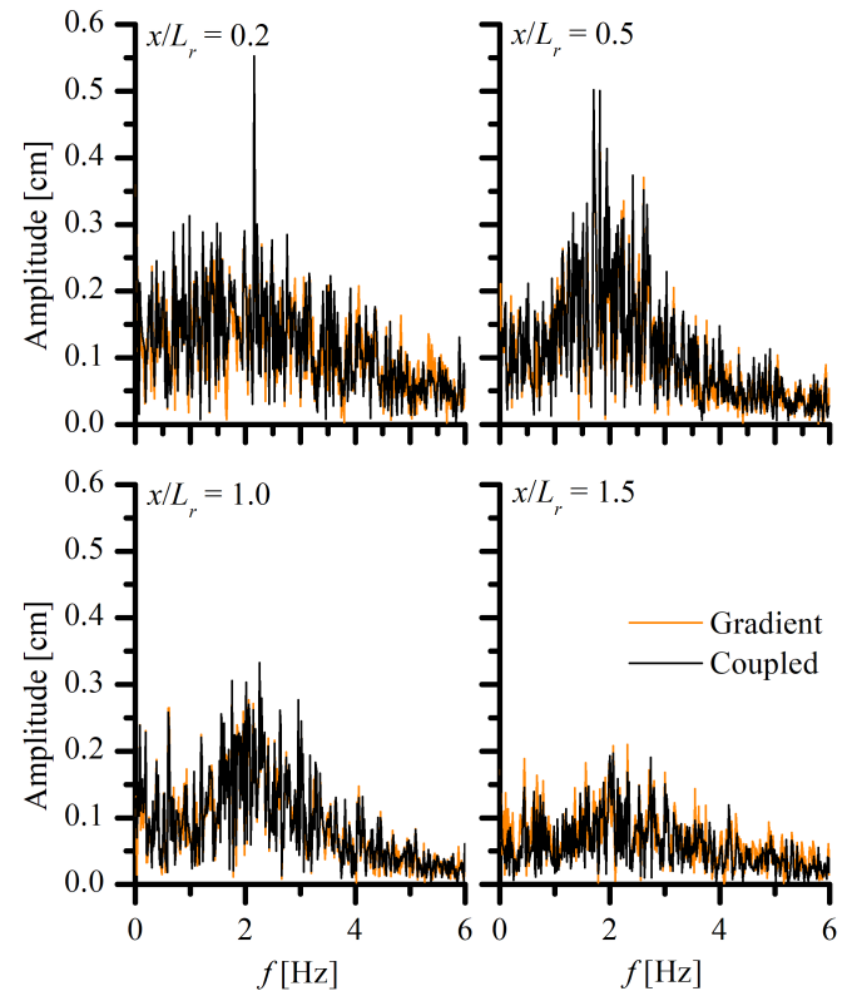

Figure 17. FFT spectrums of free-surface oscillations for four stations in the ROI, experiment 2

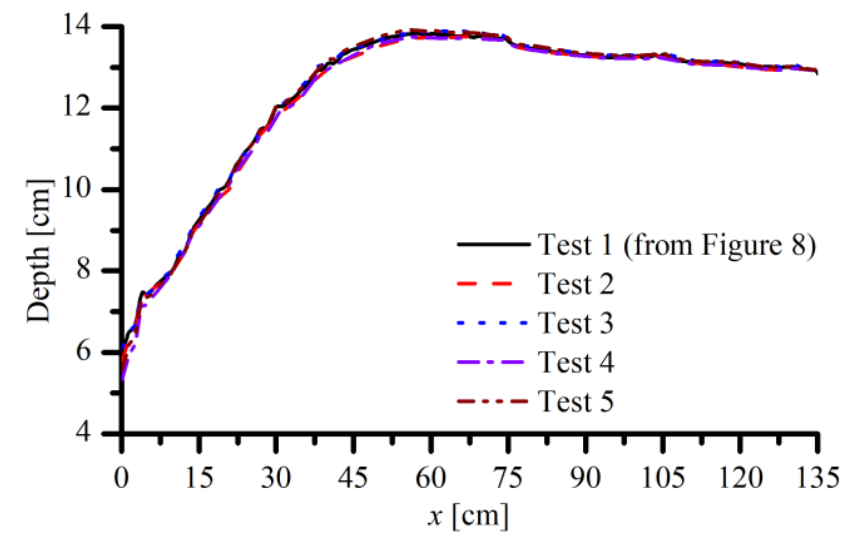

Figure 18. Time-averaged depth profiles for repeated tests, conditions of experiment 1

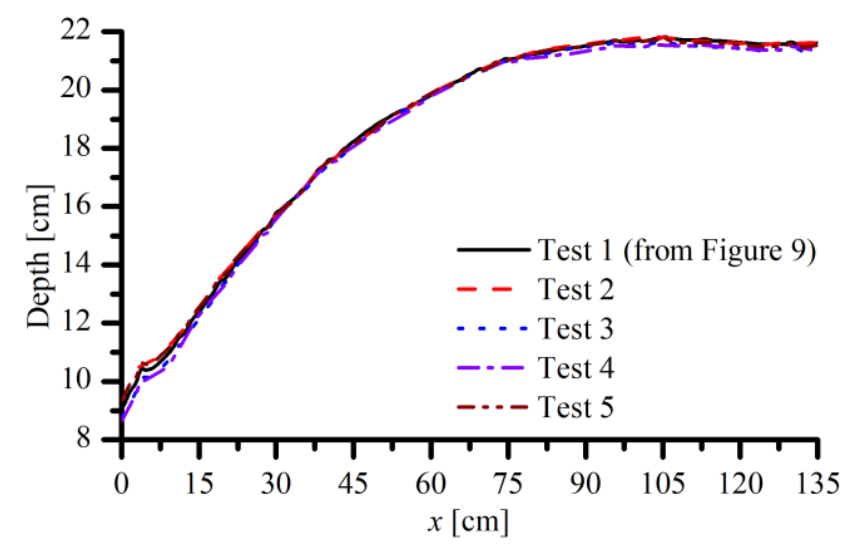

Figure 19. Time-averaged depth profiles for repeated tests, conditions of experiment 2

Table 6. Computational time structure for the coupled model, excluding I/O stages for 1800 frames

\begin{tabular}{c|l|r|r}
\multirow{2}{*}{ Exp. } & \multicolumn{1}{|c|}{ Stage } & \multicolumn{2}{c}{ Time } \\
& & {$[\mathbf{s e c}]$} & \multicolumn{1}{c}{$[\mathbf{\%}]$} \\
\hline \hline \multirow{4}{*}{1} & Gradient analysis & 2344 & 76.0 \\
& HED & 265 & 8.6 \\
& Spatial filtering & 212 & 6.9 \\
& Temporal filtering & 267 & 8.7 \\
\cline { 2 - 4 } & Total & $\mathbf{3 0 8 8}$ & $\mathbf{1 0 0}$ \\
\hline \multirow{4}{*}{2} & Gradient analysis & 2347 & 76.1 \\
& HED & 261 & 8.5 \\
& Spatial filtering & 216 & 7.0 \\
& Temporal filtering & 259 & 8.4 \\
\cline { 2 - 4 } & Total & $\mathbf{3 0 8 2}$ & $\mathbf{1 0 0}$
\end{tabular}

However, image processing approach is not without some specific shortcomings. It was found that the performance of both image processing models is severely limited in aerated high velocity flows, such as in the regions upstream of the jump toe. In those hydraulic conditions, both models are 
susceptible to severe misdetections due to the motion induced blur and low contrast. The proposed detection models should not be applied to those regions, and these have been excluded from the free-surface detection in this research. Such shortcomings could be alleviated with the use of high framerate cameras or higher shutter speeds. Both such potential improvements also imply shorter camera sensor exposure and would require even greater control of environmental lighting.

Additionally, the results obtained by the image-based models could be unrepresentative of the free-surface in the entire cross-section. The assumption of the horizontal freesurface across the section could be justified for configurations with wide prismatic spillways and stilling basins, where the near-sidewall depths can adequately represent the entire crosssection. For non-prismatic configurations, it should not be used to represent the entire cross-section. However, even in such cases, the detected depths are still important for the design of basin sidewalls. Alternatively, it can easily be argued that the results from the ADM for non-prismatic configurations would be unrepresentative for sidewall depths. Thus, the image-based free-surface detection approach is not aiming to replace some commonly used methods but could rather offer different insights for stilling basin research and design.

The most significant limitation of both methods is the computational complexity of the underlying image processing algorithms. Total amount of values processed by the algorithms is equal to sum of all of pixels in all captured images, which was 729 million for each of the experiments in this research. Total analysis time of the 60 second video (4K resolution at $29.97 \mathrm{fps}$ ) was around 47 minutes for the gradient model, and around 51 minute for the coupled model (with CUDA processing for HED stage). The overall complexity of the code was briefly examined, excluding input/output (I/O) stages such as video unpacking and geometrical transformations, as those depend mostly on the performance of the storage media. It was found that the time complexity of the code relative to the total number of pixels is around $O(n)$ for both models and using parameters presented in Table 2. For the analysis of a 1-minute video, computational time structure of the coupled model is presented in Table 6. Results show that, relative to the gradient model, the additional complexity due to the HED stage is around $8.5 \%$ which can be considered low when compared to the improvements offered by the coupled model. Future research should focus on more detailed investigation and reduction of the computational complexity of proposed methods. When the duration of the analyses is not an issue, the proposed model can be used to efficiently obtain substantial amounts of data for the investigations of hydraulic jumps, but also open channel flow in general.

Due to the high accuracy and overall robustness of the proposed approach in low aeration region, future work should also investigate a more general application for non-aerated open-channel flow where methods like LIDAR are reported to provide inadequate results. This could be significant for the investigation of transient flows in long laboratory flumes. Based on the presented results, the differences between the gradient and coupled models are likely to significantly decrease in non-aerated flow conditions.

\section{Conclusions}

Based on the experimental work presented in this paper, following conclusions can be drawn:

1. Previously developed gradient-based model for freesurface interface detection can perform satisfactory when hydraulic jumps are analysed. Some discrepancies from manually acquired visual reference values are found in the high aeration regions of the jump, i.e. and the model has a slight tendency for underestimation of the visually observed depths. Average detection error was found between $-0.7 \mathrm{~cm}$ (jump at discharge of $15.4 \mathrm{~L} / \mathrm{s}$ ), and $-0.9 \mathrm{~cm}$ (jump at discharge of $34.9 \mathrm{~L} / \mathrm{s}$ ). This indicates that the accuracy of the gradient model, relative to the visually perceived free-surface levels, increases with the decrease in surface aeration intensity,

2. Coupling of the gradient approach with a deep neural network model - holistically-nested edge detection - can provide substantial improvement in terms of free-surface detection accuracy, while reducing the potential for false detections. Relative to the visual reference values, the coupled model is significantly more accurate than the gradient model, with average errors of $-0.1 \mathrm{~cm}$, and maximal errors lower than $0.5 \mathrm{~cm}$.

3. Gradient model is more susceptible to false detections of the free-surface, in conditions of spatially varying aeration intensity,

4. The coupled model is less affected by the aeration intensity of the jump since no significant differences in detection accuracy were found between the results of two experiments. The coupled model has shown fewer false detections than the gradient model,

5. In the regions of low surface aeration, both the gradient and coupled model provide comparable results,

6. Frame-by-frame detection of free-surface/depth profiles can enable more detailed spectral analyses of hydraulic jump behaviour. Since data is collected with a high spatial resolution in the ROI, FFT spectrums can be obtained for any station along the jumps,

7. ADMs consistently indicate lower time-averaged and maximal depths than those obtained through image processing. It was discussed that this can be attributed to the penetration of the acoustic signal in the surface airwater mixture in the hydraulic jump. For stilling basin sidewall design, such underestimation can be 
unacceptable. With the decrease of the surface aeration intensity in the downstream direction, the discrepancies between ADM and image-based results were shown to decrease, when both time-averaged and maximal depths are considered,

8. Repeatability of results of the proposed coupled model is examined through repeated tests in conditions identical to those of experiments 1 and 2 . The analysis indicates significant repeatability of results for time-averaged depth profiles, with differences lower than $0.1 \mathrm{~cm}$ relative to the global time-averaged depth profile (mean profile of five tests),

9. The low cost of the equipment required for the presented image-based models can enable affordable estimation of the depth profiles in laboratory conditions - in theory, any camera can be used for the data acquisition.

However, the limitations of the proposed models must be acknowledged. The following limitations were identified in this research:

1. Particular care should be devoted to the quality of the raw data. In high velocity flows, camera sensor exposure should be short, in order to reduce the motion induced blur. Depending on local conditions, this criterion may require the use of additional, external lighting sources,

2. The proposed coupled model is currently not suitable for highly aerated high velocity flow upstream of the jump toe due to motion-induced blur in the captured images,

3. The free-surface profile was detected in a single vertical plane on the flume wall, and as such can be unrepresentative of the entire water surface in case of non-symmetrical approach conditions,

4. The algorithm is computationally expensive due to the complexity of the underlying image processing steps and the sheer amount of data to be processed.

Future work should primarily be directed towards the alleviation of aforementioned limitations. The impact of the camera parameters (resolution, aperture, shutter speed, ISO), lighting conditions, and camera position relative to the ROI, on the quality of obtained results is to be investigated. The use of high framerate cameras can expand the potential of the freesurface detection to supercritical flow upstream of the jump toe. Time complexity of the underlying image processing operations is a major obstacle, which should be thoroughly analysed in future research, for the code optimization purposes. Finally, since the proposed coupled model performed accurately in the low aeration regions of the jumps, its application can be potentially generalized for free-surface flow in laboratory flumes, especially for transient openchannel flows.

\section{Acknowledgements}

This work was supported by the Ministry of Education, Science and Technological Development of Serbia, grants TR37009 and TR37010.

Authors would like to express their gratitude to Jovan Kovačević, from the Department of Geodesy, Faculty of Civil Engineering, University of Belgrade, for his help with the least square adjustment method, and Vladimir Dmitruk, from the School of Electrical Engineering, University of Belgrade, for his help with the understanding and implementation of the holistically-nested edge detection.

\section{ORCID iDs}

Robert Ljubičić https://orcid.org/0000-0002-0218-2843 Ivana Vićanović https://orcid.org/0000-0002-8759-0933 Budo Zindović https://orcid.org/0000-0002-3309-2787 Radomir Kapor https://orcid.org/0000-0003-2523-9774

\section{References}

[1] H. Chanson and L. Toombes, "Strong interactions between free-surface aeration and turbulence in an open channel flow," Exp. Therm. Fluid Sci., vol. 27, no. 5, pp. 525-535, May 2003, doi: 10.1016/S0894-1777(02)00266-2.

[2] D. B. Bung, "Non-intrusive detection of air-water surface roughness in self-aerated chute flows," J. Hydraul. Res., vol. 51, no. 3, pp. 322-329, Jun. 2013, doi: 10.1080/00221686.2013.777373.

[3] S. Kucukali and H. Chanson, "Turbulence measurements in the bubbly flow region of hydraulic jumps," Exp. Therm. Fluid Sci., vol. 33, no. 1, pp. 41-53, 2008, doi:

10.1016/j.expthermflusci.2008.06.012.

[4] F. Murzyn and H. Chanson, "Free-surface fluctuations in hydraulic jumps: Experimental observations," Exp. Therm. Fluid Sci., vol. 33, no. 7, pp. 1055-1064, 2009, doi: 10.1016/j.expthermflusci.2009.06.003.

[5] Y. Chachereau and H. Chanson, "Free-surface fluctuations and turbulence in hydraulic jumps," Exp. Therm. Fluid Sci., vol. 35, no. 6, pp. 896-909, 2011, doi: 10.1016/j.expthermflusci.2011.01.009.

[6] J. D. Nóbrega, H. E. Schulz, and D. Z. Zhu, "Free surface detection in hydraulic jumps through image analysis and ultrasonic sensor measurements," Hydraul. Struct. Soc. Eng. challenges Extrem., no. June, pp. 1-8, 2014, doi: 10.14264/uql.2014.42.

[7] R. Li, L. Montano, and S. Felder, "LIDAR measurements of free-surface characteristics in a hydraulic jump," in Hydraulics in Water Engineering Conference, 2017, p. 9.

[8] L. Montano, R. Li, and S. Felder, "Continuous measurements of time-varying free-surface profiles in aerated hydraulic jumps with a LIDAR," Exp. Therm. Fluid Sci., vol. 93, no. January, pp. 379-397, May 2018, doi: 10.1016/j.expthermflusci.2018.01.016.

R. Li, L. Montano, K. Splinter, and S. Felder, "Opportunities of LIDAR measurements in air-water flows," in E-proceedings of the 38th IAHR World Congress, 2019.

[10] M. Kramer, H. Chanson, and S. Felder, "Can we improve 
the non-intrusive characterization of high-velocity airwater flows? Application of LIDAR technology to stepped spillways," J. Hydraul. Res., vol. 1686, 2019, doi: 10.1080/00221686.2019.1581670.

[11] M. Pfister and W. H. Hager, "Self-entrainment of air on stepped spillways," Int. J. Multiph. Flow, vol. 37, no. 2, pp. 99-107, Mar. 2011, doi:

10.1016/j.ijmultiphaseflow.2010.10.007.

[12] S. Felder and H. Chanson, "Scale effects in microscopic air-water flow properties in high-velocity free-surface flows," Exp. Therm. Fluid Sci., vol. 83, pp. 19-36, May 2017, doi: 10.1016/j.expthermflusci.2016.12.009.

[13] M. Mossa and U. Tolve, "Flow Visualization in Bubbly Two-Phase Hydraulic Jump," J. Fluids Eng., vol. 120, no. 1, p. 160, 1998, doi: 10.1115/1.2819641.

[14] J. Leandro, R. Carvalho, Y. Chachereau, and H. Chanson, "Estimating void fraction in a hydraulic jump by measurements of pixel intensity," Exp. Fluids, vol. 52, no. 5, pp. 1307-1318, 2012, doi: 10.1007/s00348-011-1257-1.

[15] S. K. Misra, M. Thomas, C. Kambhamettu, J. T. Kirby, F. Veron, and M. Brocchini, "Estimation of complex airwater interfaces from particle image velocimetry images," Exp. Fluids, vol. 40, no. 5, pp. 764-775, 2006, doi: 10.1007/s00348-006-0113-1.

[16] R. F. de Carvalho and A. T. Amador, "Physical And Numerical Investigation of the Skimming Flow Over a Stepped Spillway," in Advances in Water Resources and Hydraulic Engineering, 2009, pp. 1767-1772, doi: 10.1007/978-3-540-89465-0_304.

[17] J. M. Lennon and D. F. Hill, "Particle Image Velocity Measurements of Undular and Hydraulic Jumps," $J$. Hydraul. Eng., vol. 132, no. 12, pp. 1283-1294, 2006, doi: 10.1061/(asce)0733-9429(2006)132:12(1283).

[18] B. A. Reid, M. Gavaises, R. Shilpakar, Z. Hua, D. F. Rivas, and B. Verhaagen, "PIV Study of Aeration Efficient of Stepped Spillway System PIV Study of Aeration Efficient of Stepped Spillway System," 2017, doi: 10.1088/1757-899X/209/1/012090.

[19] J. Hong, J. Yu, and H. Hahn, "Remote Detection and Monitoring of a Water Level Using Narrow Band Channel,” J. Inf. Sci. Eng., vol. 26, pp. 71-82, 2010.

[20] T. Hies, S. B. Parasuraman, Y. Wang, R. Duester, H. S. Eikaas, and K. M. Tan, "Enhanced water-level detection by image processing," no. October 2015, 2012.

[21] K. Viriyakijja and C. Chinnarasri, "Wave Flume Measurement Using Image Analysis," Aquat. Procedia, vol. 4, no. Icwrcoe, pp. 522-531, 2015, doi: 10.1016/j.aqpro.2015.02.068.

[22] Y. Lin, Y. Lin, and J. Han, "Automatic water-level detection using single-camera images with varied poses," Measurement, vol. 127, no. May, pp. 167-174, 2018, doi: 10.1016/j.measurement.2018.05.100.

[23] L. H. Erikson and H. Hanson, "A method to extract wave tank data using video imagery and its comparison to conventional data collection techniques," Comput. Geosci., vol. 31, no. 3, pp. 371-384, 2005, doi:

10.1016/j.cageo.2004.10.006.

[24] M. Kröhnert and R. Meichsner, "Segmentation of environmental time lapse image sequences for the determination of shore lines captured by hand-held smartphone cameras," ISPRS Ann. Photogramm. Remote Sens. Spat. Inf. Sci., vol. IV-2/W4, no. 2W4, pp. 1-8, Sep. 2017, doi: 10.5194/isprs-annals-IV-2-W4-1-2017.

[25] I. Hasan, T. Hies, E. Jose, R. Duester, M. Sattler, and M.
Satzger, "An Effective Camera Based Water level recording Technology for Flood Monitoring," in INTERPAEVENT, 2016, pp. 290-295.

[26] R. Ljubičić, I. Vićanović, B. Zindović, R. Kapor, and L. Savić, "Image processing for hydraulic jump free-surface detection," in 38th IAHR World Congress, 2019, no. 1, doi: 10.3850/38WC092019-0602.

[27] P. Dollár and C. L. Zitnick, "Fast edge detection using structured forests," IEEE Trans. Pattern Anal. Mach. Intell., vol. 37, no. 8, pp. 1558-1570, 2015, doi: 10.1109/TPAMI.2014.2377715.

[28] J.-J. J. Hwang and T.-L. L. Liu, "Pixel-wise deep learning for contour detection," 3rd Int. Conf. Learn. Represent. ICLR 2015 - Work. Track Proc., vol. 1, pp. 4-5, 2015.

[29] C. Schenck and D. Fox, "Detection and Tracking of Liquids with Fully Convolutional Networks," 2016.

[30] S. Xie and Z. Tu, "Holistically-Nested Edge Detection," Int. J. Comput. Vis., vol. 125, no. 1-3, pp. 3-18, 2017, doi: 10.1007/s11263-017-1004-z.

[31] MathWorks, "Camera Calibrator," 2019. [Online]. Available:

https://www.mathworks.com/help/vision/ug/single-cameracalibrator-app.html. [Accessed: 26-Mar-2019].

[32] MathWorks, "What Is Camera Calibration?," 2019. [Online]. Available:

https://www.mathworks.com/help/vision/ug/cameracalibration.html. [Accessed: 26-Mar-2019].

[33] C. Tomasi and R. Manduchi, "Bilateral filtering for gray and color images," in Sixth International Conference on Computer Vision (IEEE Cat. No.98CH36271), 1998, pp. 839-846, doi: 10.1109/ICCV.1998.710815.

[34] I. Sobel, “An Isotropic 3x3 Image Gradient Operator," no. February 2014. 2015.

[35] S. Niklaus, "A Reimplementation of HED Using PyTorch.” 2018.

[36] Y. Jia, "Caffe: An Open Source Convolutional Architecture for Fast Feature Embedding." 2013.

[37] D. Martin, C. Fowlkes, D. Tal, and J. Malik, "A Database of Human Segmented Natural Images and its Application to Evaluating Segmentation Algorithms and Measuring Ecological Statistics," in Proc. 8th Int'l Conf. Computer Vision, 2001, vol. 2, pp. 416-423.

[38] A. Savitzky and M. J. E. Golay, "Smoothing and Differentiation of Data by Simplified Least Squares Procedures.," Anal. Chem., vol. 36, no. 8, pp. 1627-1639, Jul. 1964, doi: 10.1021/ac60214a047.

[39] W. H. Hager, R. Bremen, and N. Kawagoshi, "Classical hydraulic jump: length of roller," J. Hydraul. Res., vol. 28, no. 5, pp. 591-608, Sep. 1990, doi: 10.1080/00221689009499048.

[40] M. K. Beirami and M. R. Chamani, "Hydraulic jump in sloping channels: roller length and energy loss," Can. J. Civ. Eng., vol. 37, no. 4, pp. 535-543, 2010, doi: 10.1139/L09-175.

[41] R. M. Boes and W. H. Hager, "Two-Phase Flow Characteristics of Stepped Spillways," J. Hydraul. Eng., vol. 129, no. 9, pp. 661-670, 2003, doi: 10.1061/(ASCE)0733-9429(2003)129:9(661).

[42] R. M. Boes and W. H. Hager, "Hydraulic Design of Stepped Spillways," J. Hydraul. Eng., vol. 129, no. 9, pp. 671-679, 2003, doi: 10.1061/(ASCE)07339429(2003)129:9(671).

[43] R. Ljubičić, B. Zindović, P. Vojt, D. Pavlović, R. Kapor, and L. Savić, "Hydraulic Jumps in Adverse-Slope Stilling 
Basins for Stepped Spillways," Water, vol. 10, no. 4, p. 460, Apr. 2018, doi: 10.3390/w10040460.

[44] C. E. Kindsvater, The Hydraulic Jump in Sloping Channels. TRANSACTIONS ASCE PAPER, 1944.

[45] P. R. Wolf and C. Ghilani, "Survey Measurement Adjustments by Least Squares," in The Surveying Handbook, Boston, MA, MA: Springer US, 1995, pp. $383-$ 413.

[46] M. A. Fischler and R. C. Bolles, "Random sample consensus: a paradigm for model fitting with applications to image analysis and automated cartography," Cотmun. $A C M$, vol. 24 , no. 6 , pp. 381-395, Jun. 1981, doi: $10.1145 / 358669.358692$.

[47] A. J. Peterka, "Hydraulic design of stilling basins and energy dissipators," Water Resour. Tech. Publ., no. 25, p. 240, 1974. 